\title{
Repurposing of Berbamine Hydrochloride to Inhibit Ebola Virus by Targeting Viral Glycoprotein
}

\section{Shan Cen ( $\nabla$ shancen@imb.pumc.edu.cn )}

Institute of Medicinal Biotechnology, Chinese Academy of Medical Sciences and Peking Union Medical School

\section{Dongrong Yi}

Institute of Medicinal Biotechnology Chinese Academy of Medical Sciences

\section{Quanjie Li}

Institute of Medicinal Biotechnology Chinese Academy of Medical Sciences

\section{Xiangguo Qiu}

Public Health Agency

\section{Han Wang}

Beijing Institutes of Life Science, Chinese Academy of Sciences

\section{Zhenlong Liu}

Lady Davis Institute for Medical Research and McGill AIDS Centre, Jewish General Hospital

\section{Shihua He}

Public Health Agency of Canada

\section{Wenjun Zhu}

Public Health Agency of Canada

\section{Kai Lv Lv}

Institute of Medicinal Biotechnology, Chinese Academy of Medical Sciences and Peking Union Medical School

\section{Xiangyang Chi}

Special Pathogens Program, National Microbiology Laboratory, Public Health Agency of Canada

\section{Ling Ma}

Institute of Medicinal Biotechnology Chinese Academy of Medical Sciences

\section{Yujia Wang}

Institute of Medicinal Biotechnology, Chinese Academy of Medical Sciences and Peking Union Medical School

\section{Jing Wang}

Institute of Medicinal Biotechnology Chinese Academy of Medical Sciences

\section{Yongxin Zhang}

Institute of Medicinal Biotechnology Chinese Academy of Medical Sciences

\section{Cong Zeng}

Center for Retrovirus Research, The Ohio State University 


\section{Mingliang Liu}

Institute of Medicinal Biotechnology, Chinese Academy of Medical Sciences and Peking Union Medical School

\section{Shanlv Liu}

Center for Retrovirus Research, The Ohio State University;

\section{Chen Liang}

Lady Davis Institute for Medical Research, Jewish General Hospital, McGill University

\section{Xiaoyu Li}

Institute of Medicinal Biotechnology Chinese Academy of Medical Sciences

\section{Logan Banadyga}

PHAC https://orcid.org/0000-0001-8390-2648

\section{Yi Shi}

Institute of Microbiology, Chinese Academy of Sciences https://orcid.org/0000-0002-3053-2687

\section{George Fu Gao}

NHC Key Laboratory of Biosafety, National Institute for Viral Disease Control and Prevention, Chinese Center for Disease Control and Prevention https://orcid.org/0000-0002-3869-615X

\section{Rongtuan Lin}

McGill University

\section{Biological Sciences - Article}

Keywords: Ebola virus, entry inhibitor, primed glycoprotein, antiviral drug, berbamine hydrochloride, nature compound

Posted Date: December 2nd, 2020

DOl: https://doi.org/10.21203/rs.3.rs-111854/v1

License: (c) (1) This work is licensed under a Creative Commons Attribution 4.0 International License. Read Full License 
$1 \longdiv { \text { Repurposing of Berbamine Hydrochloride to Inhibit Ebola Virus by Targeting } }$

\section{Viral Glycoprotein}

3 Dongrong $\mathrm{Yi}^{1 \#}$, Quanjie Li ${ }^{1 \#}$, Xiangguo Qiu ${ }^{2 \#}$, Han Wang ${ }^{3}$, Zhenlong Liu ${ }^{4}$, Shihua He ${ }^{2}$, Wenjun Zhu², Kai Lv ${ }^{1}$,

4 Xiangyang $\mathrm{Chi}^{2,5}$, Ling $\mathrm{Ma}^{1}$, Yujia Wang ${ }^{1}$, Jing Wang ${ }^{1}$, Yongxin Zhang ${ }^{1}$, Cong Zeng ${ }^{6}$, Mingliang Liu ${ }^{1}$, Shanlv

5 Liu $^{6}$, Chen Liang ${ }^{4}$, Xiaoyu Li ${ }^{1}$, Logan Banadyga ${ }^{2 *}$, Yi Shi ${ }^{3,7^{*}}$, George F. Gao ${ }^{3,7}$, Rongtuan Lin ${ }^{4}$, Shan Cen $^{1^{*}}$

61 Institute of Medicinal Biotechnology, Chinese Academy of Medical Sciences and Peking Union Medical School,

$7 \quad$ Beijing 100050, China;

82 Special Pathogens Program, National Microbiology Laboratory, Public Health Agency of Canada, Winnipeg, MB R3E

93 R2, Canada

103 CAS Key Laboratory of Pathogenic Microbiology and Immunology, Institute of Microbiology, Chinese Academy of

11 Sciences, Beijing 100101, China;

124 Lady Davis Institute, Jewish General Hospital, McGill University, Montreal, Quebec, Canada;

135 Laboratory of Vaccine and Antibody Engineering, Beijing Institute of Biotechnology, Beijing, China

146 Center for Retrovirus Research, The Ohio State University, Columbus, Ohio, USA;

157 University of Chinese Academy of Sciences, Beijing 100049, China;

$16 *$ Correspondence: shancen@imb.pumc.edu.cn (S.C.); shiyi@im.ac.cn (Y.S.); logan.banadyga@canada.ca (L.B.)

17 \#These authors contributed equally to this work. 

are urgently needed to control frequent EBOV outbreaks in Africa. In this study, we report that a natural compound called berbamine hydrochloride strongly inhibits EBOV replication in vitro and in vivo. Our work further showed that berbamine hydrochloride acts by direct binding to the cleaved EBOV glycoprotein (GPCl), disrupting GPcl interaction with viral receptor NPC1, thus blocking the fusion of viral and cellular membranes. Our data support the probability of developing anti-EBOV small molecule drugs by targeting viral GPcl. More importantly, since berbamine hydrochloride has been used in clinic to treat leukopenia, it holds great promise of being quickly repurposed as an anti-EBOV drug. 
Ebola virus (EBOV) and Marburg virus (MARV) are highly pathogenic. Both viruses belong to the Filoviridae, and cause acute hemorrhagic fever in humans with high fatality rates $(1,2)$. The recent EBOV outbreak (2014-2016) in West Africa claimed over 11000 lives. However, there is currently no licensed small-molecule drug to treat or prevent EBOV infection, making EBOV a severe public health threat with the possibility of causing a global pandemic ${ }^{2}$. EBOV is a negative-sense enveloped RNA virus. The virus particle is decorated with glycoprotein (GP)(3), which is solely responsible for virus entry(4-6). GP exists as a trimer, and each monomer consists of two subunits, GP1 and GP2. Following internalization at the cell surface, EBOV transports to the late endosomes where the glycan cap sheathing the receptor-binding region of GP1 is removed by endosomal cathepsin(7). Thus primed GP (cleaved GP, GPcl) interacts with the endosomal protein Niemann-Pick C1 (NPC1) and triggers the fusion of viral and endosomal membranes(8-12). Given the crucial role of GP in viral entry, it has become a primary target for the development of anti-EBOV therapies $(13,14)$.

Indeed, a number of small molecule compounds have been tested for inhibition of EBOV GP-mediated virus entry through targeting GP and cellular proteins including cathepsin, NPC1, kinase/phosphatase, ion channel, and G protein-coupled receptor (15-23). We previously identified small peptides that structurally mimic the GPCl-binding region in NPC1 and specifically inhibit EBOV entry (24). However, one potential drawback of inhibiting a cellular target is the risk of toxicity, which often deters use of such drugs in the clinic. We now report a small molecule with potent anti-EBOV activity that is able to target the receptor-binding site in GPcl.

\section{Identification of berbamine hydrochloride as an EBOV entry inhibitor.}

We previously characterized the NPC1-binding site in GPcl, consisting of residues V79, T83, K84, F88, I113, V141, and P146 (24) (Fig. 1a). Based on this result, we designed a virtual screening strategy, as shown in Fig.1b, and systematically examined 4114 compounds from the focused small compound libraries, and ranked these compounds based on the predicted binding free energies $\left(\Delta \mathrm{G}_{\mathrm{ADV}}\right)$. A total of 334 compounds had a $\Delta G_{A D V}$ lower than $-9.0 \mathrm{kcal} / \mathrm{mol}$ (Extended Data Fig. S1) and were further visually inspected for $\mathrm{H}$-bond, $\pi-\pi$, or salt bridge interactions to discard unrealistic poses with unreasonably high score. A final list of 46 compounds (Extended Data Table. S1) were selected for further study.

We first assessed the inhibitory effect of the selected 46 compounds on infection of HIV-Luc reporter virus that was pseudotyped with EBOV GP (EBOV pseudoparticle, EBOVpp) or vesicular stomatitis virus (VSV) GP (VSV pseudoparticle, VSVpp), as we described previously (24). Of these 46 compounds, EEI-10 (Fig. 1c), EEI-33 and EEI-45 (Extended Data Fig. S2) exhibited more than 80\% inhibition of EBOVpp infection, but less than $20 \%$ reduction in VSVpp infectivity, which suggests that these three compounds selectively block EBOV GP-mediated viral entry. Such a selective inhibition was also observed for the known EBOV entry inhibitor tetrandrine (thereafter called TET) (17). Next, we determined the binding affinity of the selected compounds to the purified GPcl in vitro in the BLI binding assay. The association and dissociation data suggest that only 
a

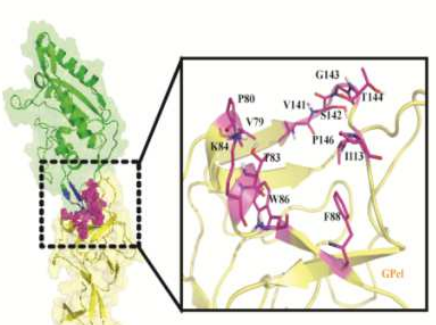

b

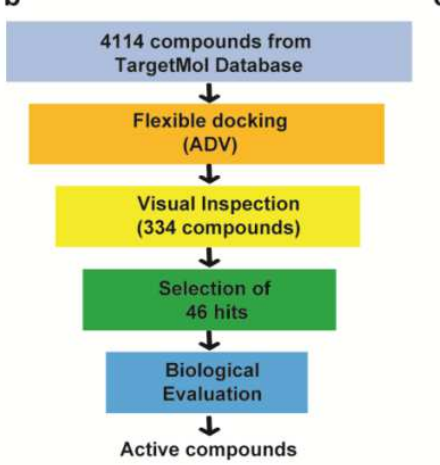

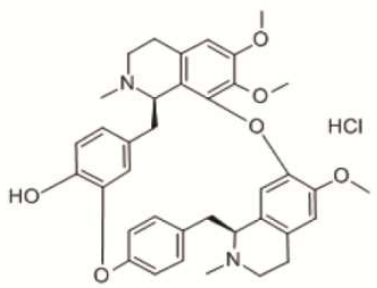

EEI-10 (Berbamine hydrochloride) MW,645.19 d

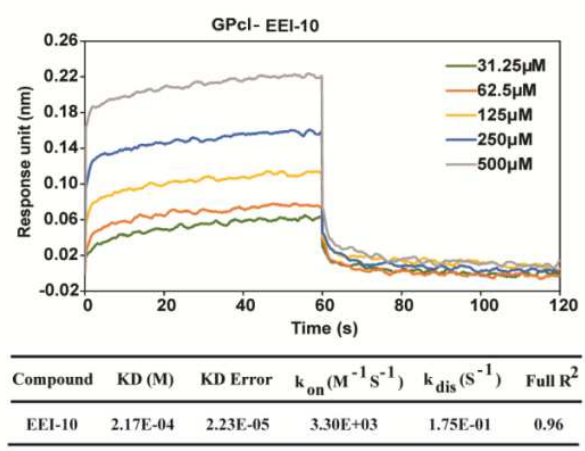

f

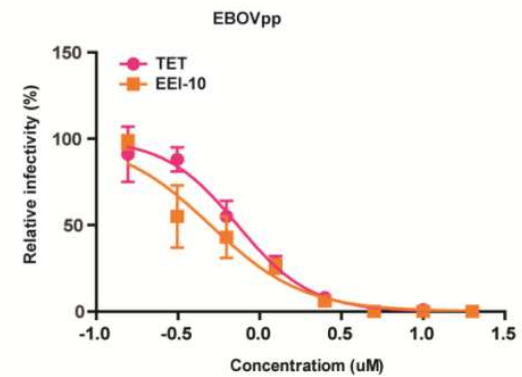

e

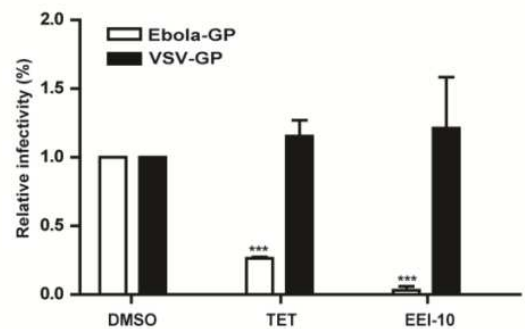

g

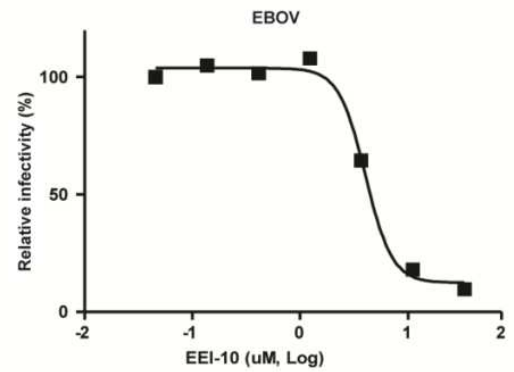

Fig.1 Identification of EEI-10 as an EBOV entry inhibitor. a, The receptor binding region identified in GPCl (PDB ID 5F1B). 
Left, the structure of the GPcl (yellow) in complex with NPC1 (green). Right, the receptor binding cavity at the top of GPcl. Highlighted in magenta is residues that constitute the interaction interface and are targeted by the virtual screening. $b$, The virtual screening strategy designed to identify small molecules targeting GPcl. c, Chemical structure of EEI-10, with molecule weight (MW). d, Representative association and dissociation sensorgrams of EEI-10 binding to EBOV-GPCl. Values of KD, KD Error, kon, kdis, and R2 are presented. e, HEK293T cells were infected with the EBOVpp in the presence of EEI-10 at the final concentration of $10 \mu \mathrm{M}$. VSVpp was used to control the specific antiviral effect of the tested drugs. Data are normalized to that of the control group (arbitrarily set as 1 ), and shown as means \pm s.d. $n=3$. f, Does-depended inhibition of EBOVpp by EEI-10 or TET. g, Inhibition of EBOV infection of Vero E6 cells by EEI-10 of different doses. EC50 and EC90 values were calculated by GraphPad Prism 5.0. Data shown are means \pm s.d. $n=3$. P values were calculated using a two-sided paired t-test.

\section{EEI-10 inhibits EBOV GP-mediated fusion of viral and cellular membranes.}

To determine whether EEI-10 inhibits EBOV GP-mediated virus entry, we first performed a time-of-addition experiment using EBOVpp. EEI-10 was added at different time points before and after EBOVpp infection. The results showed that both EEI-10 and TET markedly inhibited viral infection at the early time points of drug addition, and their inhibitory effects decreased after 4 hpi (Fig. 2a). In contrast, the HIV-1 reverse transcriptase inhibitor efavirenz (EFV) maintained its inhibitory effect up to $8 \mathrm{hpi}$. We further measured the possible effect of EEI-10 on the internalization of EBOVpp from the cell surface by quantifying the uptake of viral genomic RNA, and observed no effect as opposed to the marked inhibition by heparin (Fig. 2b), which has been shown to block the early attachment of EBOV to the target cells (26). These data support that EEI-10 inhibits an early step of EBOVpp infection, after EBOV GP-mediated internalization from the cell surface but prior to viral RNA replication.

It is known that following internalization at the cell surface, EBOV particles translocate to the acidic late endosomes where virus-cell membrane fusion occurs, leading to the release of viral RNA into the cytoplasm $(27,28)$. Imaging EBOVpp-infected cell at $4 \mathrm{hpi}$ revealed a similar number of virions in the LAMP1 (lysosomal associated membrane protein 1)-positive late endosome/lysosome compartments in cells treated with either EEI-10 or control DMSO (Fig. 2c and Extended Data Fig. S5). This further supports the data in Figure $2 b$ that EEI-10 does not affect the early events of virus entry from uptake of virus at the cell surface to trafficking of virions to the acidic compartments. We next tested whether the fusion of viral and cellular membranes is inhibited by EEI-10 using a beta-lactamase-Vpr chimeric protein (BlaM-Vpr) release assay that has been established to measure the delivery of HIV-1 core into the cytoplasm(29). We found that EEI-10 significantly reduced the cytoplasmic entry of BlaM-Vpr from EBOVpp by approximate 80\%, whereas no such effect of EEI-10 was observed for VSVpp (Fig. 2d and Extended Data Fig. S6). This suggests that EEI-10 specifically inhibits the delivery of BlaM-Vpr into the cytoplasm resulting from EBOV GP-mediated membrane fusion. In further support of this conclusion, adding MG132 to cell culture for preventing the degradation of incoming virion, a pronounced increase was detected in the level of EBOVpp particles in 

Fig. S7), suggesting that EEI-10-mediated delay of virus entry at the late endosomes causes accumulation of EBOVpp particles.

a

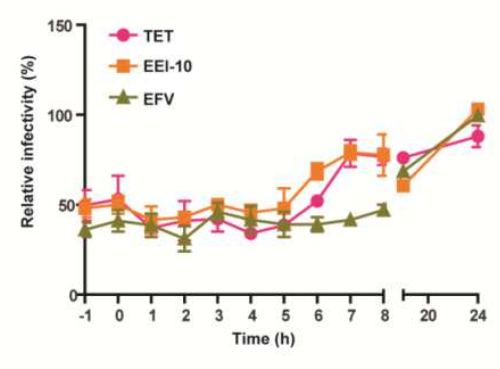

d

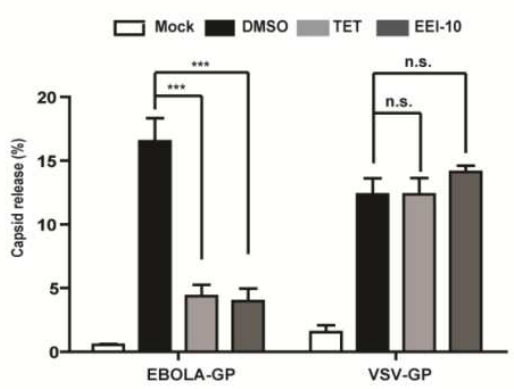

b

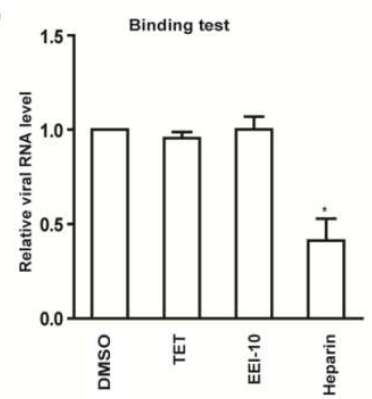

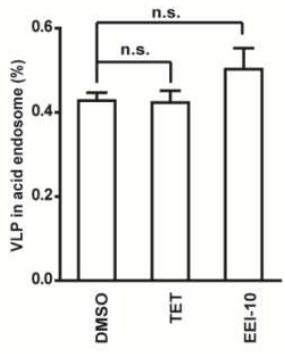

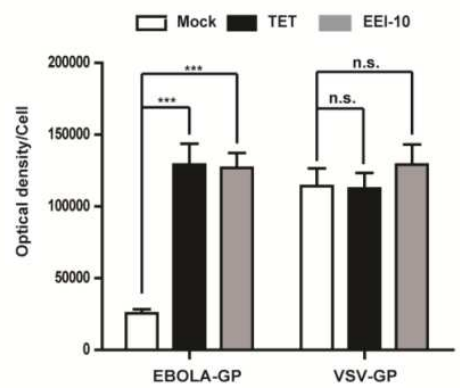

Fig. 2 EEI-10 inhibits EBOV-GP dependent virus-cell membrane fusion. a, Time-addition experiment to determine the inhibition mechanism by EEI-10, TET and efavirenz (EFV). Data shown are the means \pm s.d. $n=3$. $b$, Binding test to measure the effect of EEI-10 on virus binding to the cell membrane. Heparin was used as a positive control. Viral RNA from virus particles binding to the cell membrane was quantified by qRT-PCR. Data are normalized to that the control group which is arbitrarily set to 1. c, Virus particles (red) in the acidic endosomes were scored by their colocalization with Lamp1 (green) (as for fig. S5). Fluorescence intensity of green and red signals from randomly selected cells ( $\mathrm{n} \geq 30$ ) was quantified by Image-Pro Plus 10 software. d, VLPs (EBOVpp and VSVpp) loaded with BlaM-Vpr were used to measure membrane fusion and virus capsid release into the cytoplasm after EEI-10 and TET treatment by flow cytometry (as for fig. S6). The bar graph presents the percentages of virus release into the cytoplasm. Data shown are the means \pm s.d. $n=$ 3. e, VLPS (EBOVpp and VSVpp) accumulated in the cytoplasm after EEI-10 and TET treatment. The number of VLPs (red signals) in confocal images was determined from randomly selected cells ( $\mathrm{n} \geq 80$ ). The fluorescence intensity was quantified by Image-Pro Plus 10 software. P values were calculated using a two-sided paired t-test.

\section{EEI-10 disrupts binding of EBOV GPCl to NPC1.}

140 We next determined whether EEI-10 inhibits EBOV GP-mediated entry by disrupting the binding of GP to its receptor NPC1 in the infected cell. We first observed that that EEI-10 treatment reduced the colocalization of EBOVpp particles with NPC1 in the infected cells (Fig. 3a), as opposed to TET which targets the two-pore channels and had no effect as previously reported (17). EBOV GP can only bind to NPC1 after cleavage by the endosomal cathepsin. We thus pretreated EBOVpp with the protease thermolysin, which completes GP 
cleavage(8), and then infected cells in the presence of EEl-10. Again, strong inhibition was observed with EEI-10 but not with cysteine protease inhibitor E-64 (Fig. 3b). Thus, EEI-10 diminishes the colocalization of EBOVpp with NPC1 not through affecting GP proteolysis. We next performed co-immunoprecipitation to measure the association of GPCl with its receptor NPC1 and found that EEI-10, but not TET, markedly reduced GPCl-NPC1 interaction (Fig. 3c). This observation was supported by the data of proximity ligation assay (PLA), showing that treatment with EEI-10 diminished GPCI-NPC1 interaction in the infected cells by approximately $80 \%$ (Fig. 3d). We further showed that EEI-10 did not affect the cellular content of cholesterol (Extended Data Fig. S8) in contrast with NPC1 inhibitors U18666A and compound 3.0 that inhibited EBOV entry by causing cholesterol accumulation in late endosomes, suggesting that EEI-10 does not act on NPC1. We thus conclude that EEI-10 binds to GPcl, prevents GPCl from binding NPC1, and inhibits GP-mediated fusion of viral and cellular membranes.

a
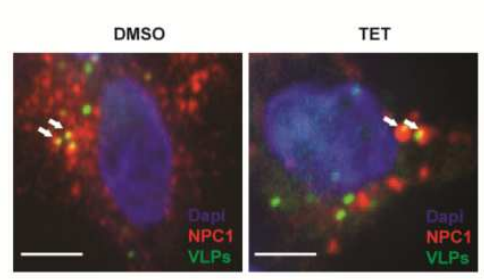

c

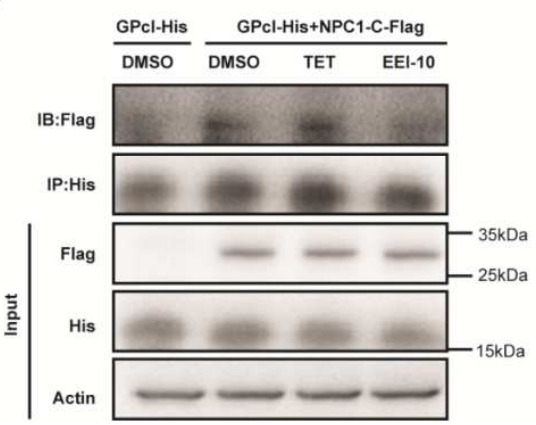

EEl-10

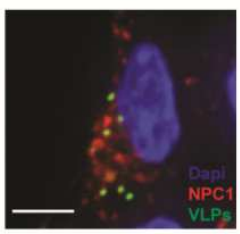

d b

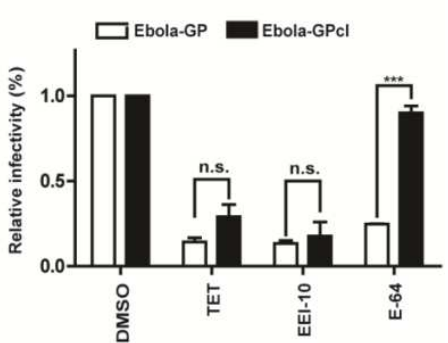

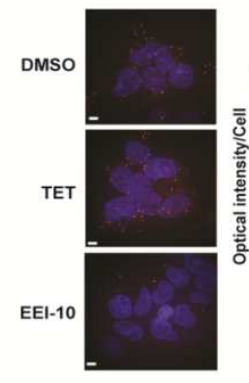

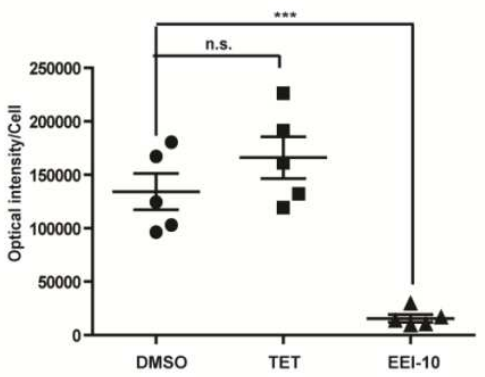

Fig. 3 EEI-10 disrupts the binding of EBOV-GPCl to NPC1. a, Colocalization of Ebola VLPs with NPC1 was determined by incubating VLPs with HEK293 cells for 4 hours. Representative confocal images are shown. Colocalized Ebola VLPs (Green) and NPC1(Red) were indicated by arrowheads. Scale bars, $5 \mu \mathrm{m}$. b, HEK293T cells were exposed to EBOVpp which were treated with protease thermolysin to generate EBVO GPcl, in the presence of EEI-10 or TET. The cysteine protease inhibitor E-64 was used as a positive control. Luciferase activities were measured and normalized to those of untreated controls (mean \pm s.d. $n=3$ ). Each data set is representative of three independent experiments. c, Interaction between NPC1-C-Flag and GPcl-His was determined with Co-IP in the presence of EEI-10 or TET. Data shown are the representative of three independent experiments. $d$, In situ PLA to analyze the interaction between Ebola-GP (or VSV-GP) and cellular NPC1 in the presence of the EEI-10 or TET. Representative confocal images are shown. Scale bars, $5 \mu \mathrm{m}$. Mean fluorescence intensity was collected from at least 30 cells and further analyzed for each treatment.

GPS of EBOV and MARV share a similar binding pocket for EEI-10 and are both inhibited by EEI-10. 
We next used the AutoDock Vian computation program to model the binding of EEI-10 to GPcl. In the generated model of GPCl in complex with EEI-10 (Fig. 4a), EEI-10 is located at the hydrophobic pocket in GPcl and forms hydrogen bond interaction with the amino residue E112. We further employed the MM/GBSA computation approach to calculate the binding free energy of EEI-10 and decomposed the free energy at the amino acid level. The cavity analysis showed that the major contributing amino acids to the binding of EEI-10 include E112, T83, I113, V141, F88, L111, W86, V79, P80, and G143, which are ranked based on their $\Delta \mathrm{G}_{\mathrm{ADV}}$ in the range from -2.54 to $-0.62 \mathrm{kcal} / \mathrm{mol}$ (Extended Data Table. S2).

To validate this binding model, we mutated the three top-ranking amino acids E112, T83 and I113, and examined their effect on the sensitivity of EBOV GP-mediated entry to EEI-10. The E112A mutation severely impaired viral infectivity, thus was not further tested for EEl-10 inhibition (Extended Data Fig. S9). The I113A GP mutant was refractory to EEI-10 while remaining sensitive to the TET entry inhibitor (Fig. $4 b$ ). In the meantime, the T83A mutation exerted no effect on the sensitivity of GP to either EEI-10 or TET inhibition (Fig. 4b); this is likely because A83 is able to restore the van der Waals interaction with EEI-10 which is observed for T83 as predicted in our computation model (Extended Data Table. 2). These data demonstrate that I113 is a key residue in contact with EEI-10 and that its mutation leads to resistance to EEI-10, which supports the structural model of GPcl binding to EEI-10.

Recent studies have revealed a common receptor-binding site in GPs of several filoviruses including EBOV and MARV $(13,30)$, which suggests that EEI-10 may inhibit MARV infection as well. Indeed, EEI-10 inhibited the MARV-GP/HIV-luc virus infection at an EC50 $=0.99 \mu \mathrm{M}$, and inhibited MARV itself at an EC50 $=4.06 \mu \mathrm{M}$ (Fig. 4c, d). We further conducted computational docking of EEI-10 to MARV-GP1 and then preformed MD simulation to relax the complex. The binding free energy of the MARV GP/EEI-10 was calculated as

$193 \Delta \mathrm{G}_{\mathrm{ADV}}=-9.3 \mathrm{kcal} / \mathrm{mol}$, which is moderately higher than that of EBOV-GPCl$/ \mathrm{EEl}-10$ complex $\left(\Delta \mathrm{G}_{\mathrm{ADV}}=-10.9\right.$ $\mathrm{kcal} / \mathrm{mol}$ ). Notably, these two complexes are well superposed at the EEl-10 binding sites (Fig. 4e). The top 10 amino acids in MARV-GP1 that contribute most to the binding energies are L64, S67, K68, A71, F72, 195, V97, 1125, G127, and N129 (Extended Data Table. S3), which are equivalent to P80, T83, K84, G87, F88, L111, I113,

197 V141, G143, and G145 in EBOV GPcl. Importantly, seven of the top 10 major contributing amino residues are 198 shared in both complexes (Fig. 4f). These data suggest that EEI-10 is able to bind to the GPs of EBOV and 199 MARV, and inhibit both viruses. 
a
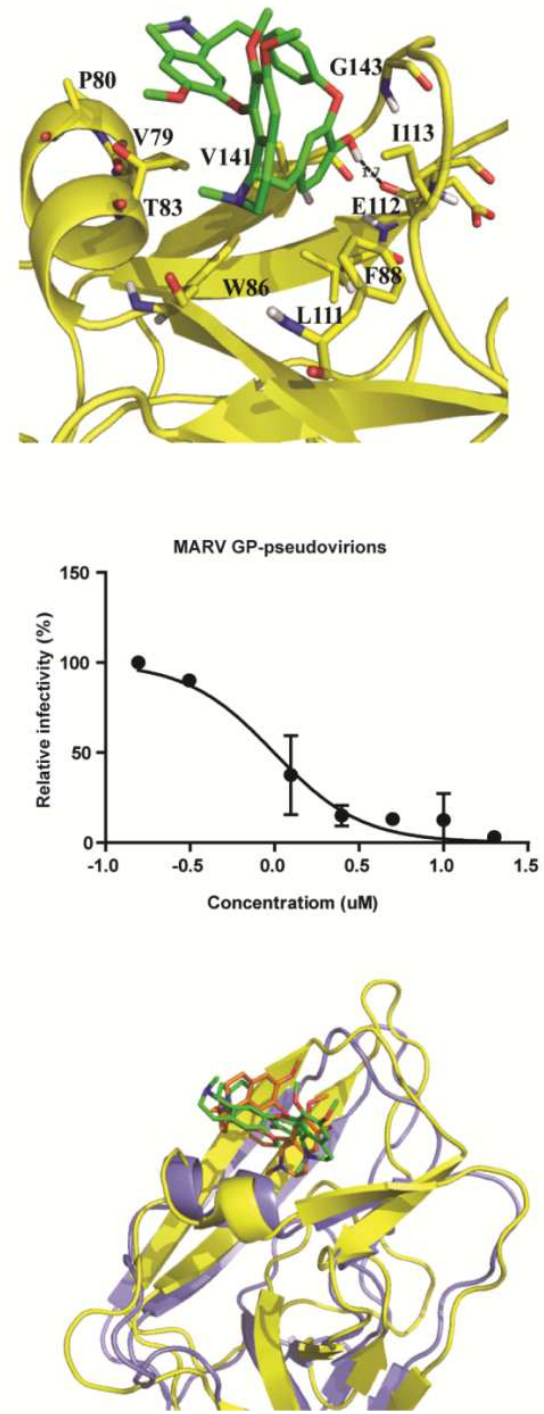

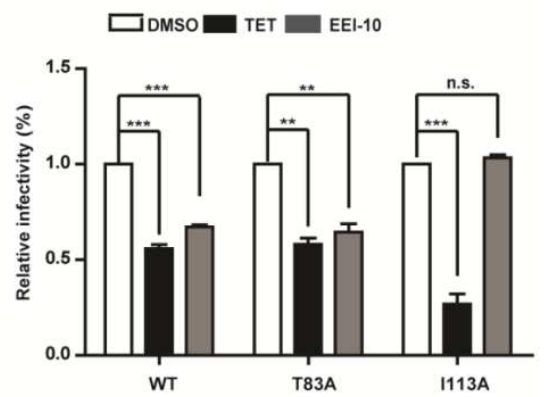

d

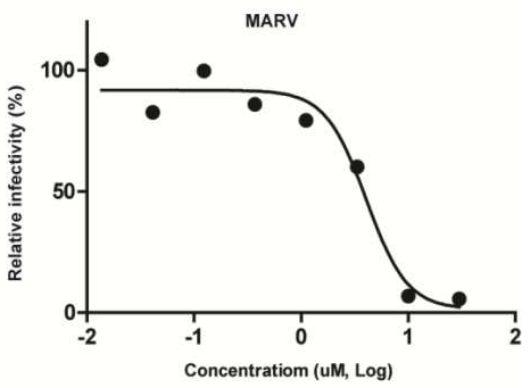

f

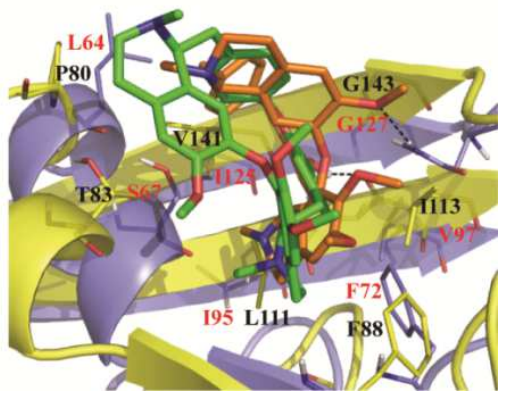

Fig. 4 EEI-10 inhibits infection mediated by the GPs of EBOV and MARV. a, Structural model of EEI-10 binding to GPcl. Amino residues (in yellow) binding to EEI-10 (in green) are shown as sticks. The oxygen, nitrogen, and hydrogen atoms are colored in red, blue, and white, respectively. For clarity, only polar hydrogen atoms are shown. The PDB ID of the EBOV-GPcl crystal structure used for molecular modeling is 5F1B. b, Inhibition of the T83A and I113A GP mutants by EEI-10. Luciferase activities were measured and normalized to those of untreated controls. Data shown are the average of three independent experiments, presented in mean \pm SD. c, Inhibition of MARV GP-pseudovirions by EEI-10 of different doses. $d$, Different doses of EEI-10 were tested for inhibition of MARV infection in Vero E6 cells. EC50 values were calculated with GraphPad Prism 5.0. Each data point is the average of three independent experiments. e, Superposition of the structures of the EBOV-GPCl/EEI-10 and MARV-GP1/EEI-10 complexes. EBOV-GPCl and MARV-GP1 are shown as yellow and slate cartoons, and their associate inhibitor EEI-10 was shown as green and orange sticks. $\mathrm{f}$, Comparison of protein-ligand interactions in the EBOV-GPCl/EEI-10 and MARV-GP1/EEI-10 complexes. Key residues in the MARV-GP1/EEI-10 binding pocket are highlighted in red. The PDB IDs of crystal structures used for molecular modeling are 5F1B for EBOV GPcl and 5UQY for MARV GP1. 
Finally, we asked whether EEI-10 was able to protect mice from lethal EBOV infection. Mice were treated orally once per day for 6 days with $100 \mathrm{mg} / \mathrm{kg} \mathrm{EEl-10} \mathrm{beginning} \mathrm{the} \mathrm{day} \mathrm{prior} \mathrm{to} \mathrm{inoculation} \mathrm{with} \mathrm{a} \mathrm{lethal}$ dose of mouse-adapted EBOV (MA-EBOV) (Fig. 5a). Remarkably, $100 \%$ of the mice treated with EEI-10 survived infection while only one of the PBS-treated animals survived (Fig. 5b), although all exhibited weight loss (Fig. 5c). To determine whether EEI-10 was also effective if treatment was begun post-infection, mice were again inoculated with MA-EBOV and then treated orally with the same dose of drug beginning at 1 day post-infection for a period of 6 days. Whereas all PBS-treated mice succumbed to infection by day 7, nearly all mice treated with EEI-10 survived, and the time to death was prolonged in the single treated animal that died (Fig. 5d). Again, all animals exhibited weight loss (Fig. 5e), suggesting that, while EEl-10 treatment provided a significant survival advantage, it did not prevent some signs of disease.

\section{a}

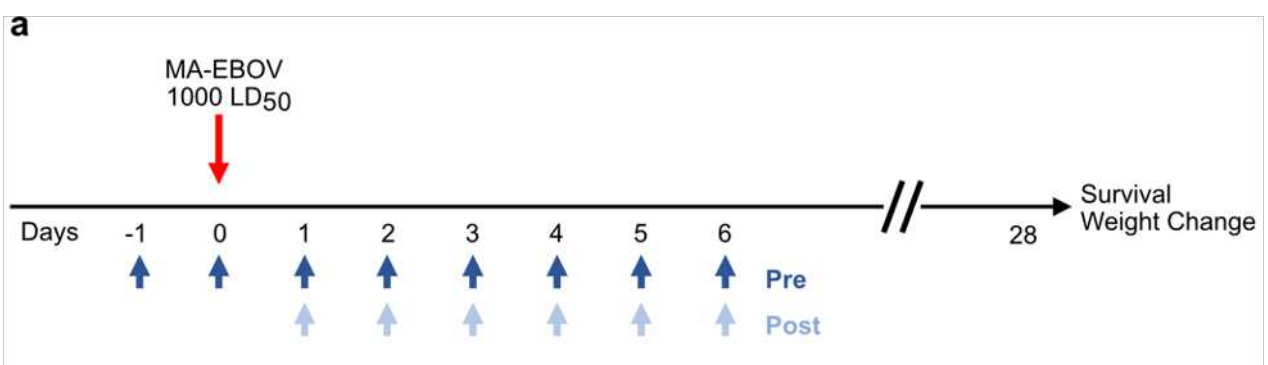

b
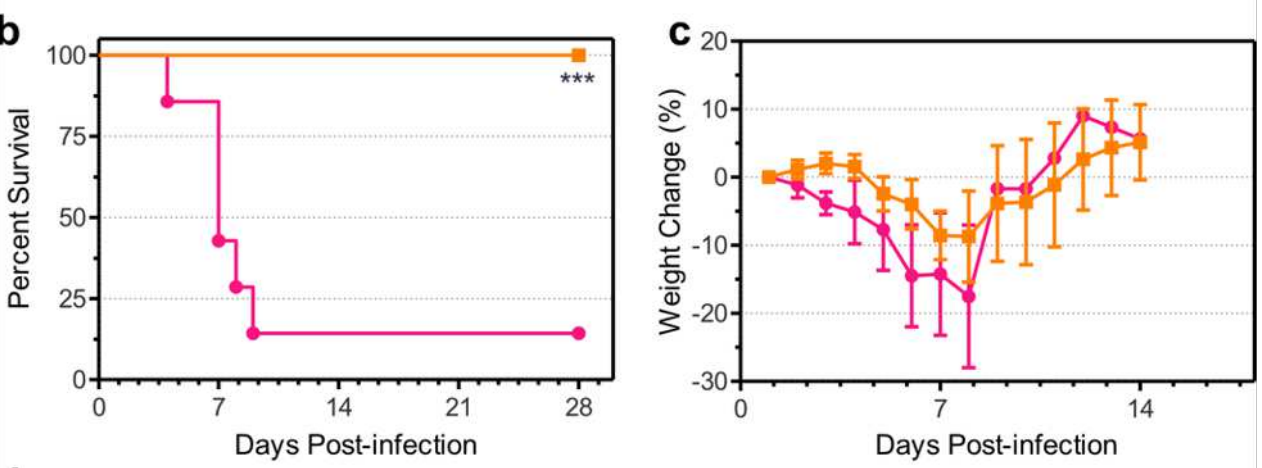

d
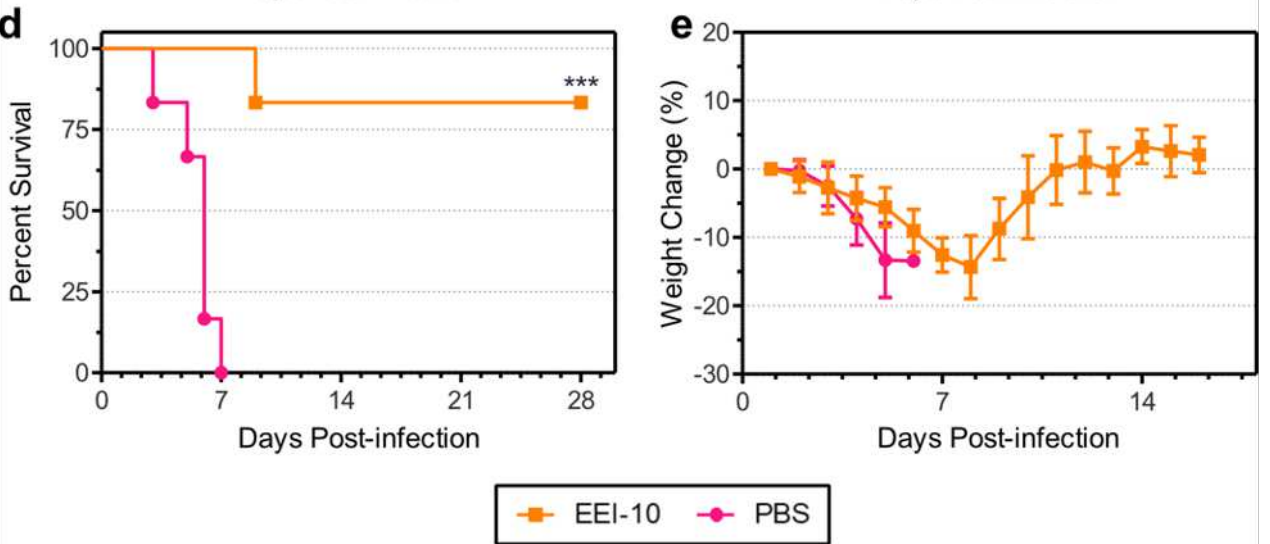

Fig. 5. EEI-10 prevents lethal EBOV infection in mice. a, Schematic representation of EEI-10 treatment protocol and EBOV infection of mice. b,c, Balb/c mice were treated orally with EEI-10 $(100 \mathrm{mg} / \mathrm{kg} ; n=10)$ or PBS $(n=7)$ one day before infection with 1000 LD50 mouse-adapted EBOV (MA-EBOV). Treatment continued once daily until day 6 after infection. 
Survival (in b) and average percent weight change \pm standard deviation (in c) are shown. $d, e$, Balb/c mice were infected with 1000 LD50 MA-EBOV one day prior to oral treatment with EEI-10 $(100 \mathrm{mg} / \mathrm{kg} ; \mathrm{n}=6)$ or PBS $(n=6)$. Treatment continued once daily until day 6 post-infection. Survival (in d) and average percent weight change \pm standard deviation (in e) are shown Survival curves were compared using the Log-rank Test; ${ }^{* *}, p \leq 0.001$.

This remarkable protective efficacy of EEI-10 may be partially attributed to the relatively weak interaction of GPcl with its receptor NPC1. With a KD of $158 \mu \mathrm{M}$, as measured in vitro using recombinant proteins (31), the interaction between these two proteins was likely easily disrupted by EEI-10. Through medicinal chemistry to modify EEI-10 and select for variants with greater affinity to GPcl, we expect to identify EEI-10 derivatives exhibiting much stronger inhibition of EBOV as well as a higher genetic barrier to deter the development of viral resistance. Additionally, as a drug to treat leukopenia, EEI-10 is expected to modulate certain cellular pathways and host immunity, which may have also contributed to its protection of mice against lethal EBOV infection.

Of note, EEl-10 was demonstrated to bind to EBOV GPcl and potently inhibit virus entry, whereas in vitro binding assay revealed that EEI-10 exhibited a low affinity for GPcl, with KD values above $100 \mu \mathrm{M}$ (Fig. 1d), likely due to the inability of the molecule to fit into and fully fill the extremely large receptor binding cavity (Fig. 1a). Nevertheless, a similar affinity $(K D=158 \mu \mathrm{M})$ was reported in the complex structure of GPcl bound to NPC1(31), suggesting the feasibility of EEI-10 to compete with NPC1 for binding to GPcl. Moreover, lower $\mathrm{pH}$ was reported to enhance the interaction between GPcl and NPC1(9). This implies that the binding affinity of EEI-10 in the acidic endosome where it occurs may be underrated in vitro.

In summary, we have identified a natural compound berbamine hydrochloride (EEI-10) that potently inhibits EBOV infection of cultured cells through direct binding to viral GPcl and impairing the interaction of GPCl with the viral receptor NPC1. Our data further showed that EEI-10 strongly inhibits another pathogenic filovirus, MARV, implicating the potential use of EEI-10 as a broad-spectrum inhibitor of filovirus infection. Given that berbamine hydrochloride is already approved to treat leukopenia and has been thoroughly evaluated for its safety and pharmacokinetics properties, this drug holds great promise for saving the lives of EBOV patients and preventing future EBOV epidemics or pandemics. 
Author Contributions: D.Y. executed all the in vitro antiviral testing against pesudotyped virus. Q.L. performed the screening, predicted the binding mode of compounds, and wrote the original draft. X.Q., S.H., W.Z. and X.C. conducted in vivo efficacy studies and performed associated sample analyses. H.W. purified the GP protein. Y.W. conducted cell-based assays for cytotoxicity. C.Z. and S.L. participated in the Co-IP experiment. K.L. and M.L. were responsible for the synthesis and characterization of small molecules. L.M. and J.W. established the EBOVpp assay, Y.Z. conducted statistical analysis. X.L., C.L., L.B., and S.C. oversaw the analysis and revised the manuscript. S.C., R.L., G.G., Y.S., Z.L. and L.B. conceived and supervised the project, evaluated results and approved the manuscript.

Funding: This research was funded by the National Mega-project for Innovative Drugs (Grant No. 2018ZX09711003-002-002), the CAMS Innovation Fund for Medical Sciences (Grant No. CAMS-I2M-1-012), the National Natural Science Foundation of China (Grant No. 81802019 and 81902075), the Beijing Natural Science Foundation (Grant No. 7184228), the Peking Union Medical College Youth Fund (Grant No. 3332016063 and 3332018096 ), the China Ministry of Science and Technology National 973 Project (Grant No. 2014CB542503), the Excellent Young Scientist Program from the NSFC (Grant No. 81622031), and the National Key Research and Development program of China (Grant No. 2016YFD0500307),

Conflicts of Interest: The authors declare no conflict of interest.

\section{Methods}

\section{Cell lines, Plasmids and Virus}

HEK293T, Vero E6 and HeLa cells were obtained from the American Type Culture Collection and maintained at $37^{\circ} \mathrm{C}$ and $5 \% \mathrm{CO}_{2}$ in DMEM supplemented with $10 \% \mathrm{HI}-\mathrm{FBS}, 2 \mathrm{mM}$ L-glutamine, $50 \mathrm{U} / \mathrm{ml}$ penicillin, and 50 $\mathrm{ug} / \mathrm{ml}$ streptomycin. Plasmids encoding EBOV-GP, vesicular stomatitis virus envelope protein (VSV-GP) and HIV-I luciferase reporter vector pNL4-3.Luc.R-E- were kept in the Institute of Medicinal Biotechnology, Beijing. The GP genes of MARV (GenBank Accession No. ABA87127.1, amino acid (а.а.) 5941-7986) were synthesized by Suzhou GENEWIZ Biotechnology Co., Ltd., and inserted into pcDNA3.1(+) vector (Invitrogen). Plasmids encoding Vpr-Blam was kindly provided by Dr. Xu Tan (Tsinghua University, China). All work with recombinant EBOV expressing EGFP (Ebola virus H.Sapiens-rec/GIN/2014/Makona-Guekedou-C07-EGFP), mouse-adapted EBOV variant Mayinga(32) and wild type MARV variant Angola (Marburg virus H.spaiens-tc/AGO/2005/Angola) was performed in the containment level 4 laboratories at the Canadian Science Centre for Human and Animal Health (CSCHAH), Public Health Agency of Canada, Winnipeg, MB, Canada.

\section{Compounds and antibodies}

TargetMol's small-compound library subsets (anti-virus compound library, anti-infection compound library, nature compound library, plant-sourced compound library, anti-inflammation library, and inhibitors library) were used for virtual screening. Small molecules selected from docking procedure were purchased from 
Target Molecule Corp. Tetrandrine (TET) and efavirenz (EFV) were maintained in the Institute of Medicinal Biotechnology, Beijing.. The purity of all tested compounds is more than $95 \%$. The following antibodies were used: Mouse anti-His (TA-02; ZSGB-Bio), Mouse anti-Flag (8146S; CST), rabbit anti-Flag (B1020; Biodragon), Mouse anti-NPC1 (ab134113; Abcam), Mouse anti-p24 (ab9071; Abcam), Rabbit anti-p24 (produced by our own lab), Mouse anti-Lamp1 (ab24170; Abcam), Mouse anti- $\beta$-actin monoclonal antibody (Abcam) and goat anti-rabbit secondary antibody (Beijing zhongshan jinqiao Biotechnology). Alexa Fluor-conjugated secondary antibodies were purchased from Life Technologies.

\section{Generation of recombinant HIV pseudotyped with virus glycoproteins}

306

307

Ebola pseudotyped viruses (EBOV-GP/HIV-luc) were produced as previously described(24). Pseudotyped viruses bearing VSV envelope protein (VSV-G/HIV-luc) and MARV envelope protein (MARV-GP/HIV-luc) were produced in a similar way.

\section{Infection assay}

Screening of the selected compounds using pesudotyped virus was performed in 96-well plates as previous described(24). Briefly, cells were infected with the EBOV-GP/HIV-luc in the presence of compounds at a final concentration of $10 \mu \mathrm{M}$. The infected cells were lysed and the anti-EBOV activity of test compounds was evaluated by measuring the luciferase activities at $48 \mathrm{~h}$ post infection (hpi). DMSO and TET were used as negative and positive controls, respectively. The VSV-G/HIV-luc was used as a control to determine the specificity of compounds. The $50 \%$ effective concentration (EC50) and EC90 values were calculated by using a four-parameter logistic regression in Prism 5 (GraphPad).

To analyze the retention of EBOVpp virions in newly infected cells, HEK293T cells were infected with EBOV-GP/HIV-luc at an $\mathrm{MOI}$ of 1.0 in the presence of MG132 $(4 \mu \mathrm{M})$. After $4 \mathrm{~h}$, cells were fixed in $4 \%$ paraformaldehyde for $30 \mathrm{~min}$ on ice and virus was stained with rabbit or mouse anti-p24 antibody, followed by an anti-Rabbit AlexaFluor-555 antibody or anti-mouse AlexaFluor-488 antibody. Fluorescence was monitored with a confocal microscope with a $100 \times$ oil objective lens.

To assess the effect of berbamine hydrochloride on EBOV and MARV replication, Vero E6 cells were pretreated with drug (0 to $200 \mu \mathrm{M})$ or DMSO for $1 \mathrm{~h}$ at $37^{\circ} \mathrm{C}$ and infected at a multiplicity of infection (MOI) of 0.1 with EBOV expressing enhanced green fluorescent protein (EBOV-EGFP) or wild type MARV for $1 \mathrm{~h}$ at $37^{\circ} \mathrm{C}$, after which the inoculum was removed and replaced with fresh medium (DMEM plus $2 \%$ FBS). Cells were further incubated for $72 \mathrm{~h}$ in the presence of EEI-10 or DMSO. For EBOV-EGFP-infected cells fluorescence was quantified on a Biotek Synergy HTX plate reader. The percent relative infectivity was determined by comparing fluorescence readings of EEI-10-treated cells to those of DMSO-treated control cells. For MARV-infected cells, supernatants were harvested and viral RNA was quantified, as described below. The percent relative infectivity was determined by comparing the amount of viral RNA detected in EEI-10 treated cells to that of DMSO-treated control cells. 
For HIV-based infections, total RNA was extracted from infected cells by use of an RNA extraction kit (Tiandz). The level of viral RNA was determined by performing qRT-PCR analysis by use of a one-step SYBR PrimeScript RT-PCR kit. The primer pair (5'-TTAAGCCTCAATAAAGCTTGCC-3' and 5'-GTTCGGGCGCCACTGCTAGA-3') amplifies the LTR of HIV. Levels of cellular glyceraldehyde-3-phosphate dehydrogenase (GAPDH) RNA were amplified with primers 5'-ATCATCCCTGCCTCTACTGG-3' and 5'-GTCAGGTCCACCACTGACAC-3'; the results served as an internal control to normalize the level of HIV RNA.

For MARV infections, viral RNA was extracted from tissue culture supernatants using the QIAamp viral RNA minikit (Qiagen). Viral RNA levels were quantified by qRT-PCR using the LightCycler 480 thermal cycler (Roche) and the LightCycler 480 RNA Master Hydrolysis Probes kit (Roche) along with the following primers and probe: 1-F, 5'-GCAAAAGCATTCCCTAGTAACATGA-3'; 1-R, 5'-CACCCCTCACTATRGCGTTYTC-3'; 2-F, 5'-GCGAAGGCATTCCCTAGTAATATGA-3'; 2-R, 5'-CACCTCTTACTATGGCATTCTC-3'; probe, 5'-56-FAM/TGGCAC CAY/ZEN/AATTCAGCAAGCATAGG/ 3IABkFQ-3'. Cycling conditions were as follows: $63^{\circ} \mathrm{C}$ for 3 min and $95^{\circ} \mathrm{C}$ for $30 \mathrm{~s}$, then 45 cycles of $95^{\circ} \mathrm{C}$ for $15 \mathrm{~s}$ and $60^{\circ} \mathrm{C}$ for $30 \mathrm{~s}$.

\section{Cytotoxicity analysis}

HEK293T and Vero E6 cells were seeded in 96-well plates. Following overnight incubation at $37^{\circ} \mathrm{C}$, Tetrandrine and Berbamine hydrochloride were added at different concentrations used for the viral infection studies. Cells in culture media without drug served as control. At 48h, Cell Counting kit-8 (CCK-8, Beyotime, China) was used to evaluate cell viability.

\section{Blam-Vpr releasing assay}

355

For production of pseudotyped viruses incorporating Blam-Vpr, HEK293T cells were transfected with $6 \mu \mathrm{g}$ of pNL4-3.Luc.R-E-, $6 \mu \mathrm{g}$ of pEbola-GP or pVSV-GP, $3 \mu \mathrm{g}$ of pBlam-Vpr and $1.5 \mu \mathrm{g}$ of pAdvantage using Lipo2000 in $10-\mathrm{cm}$ dishes. Culture supernatants were collected after $48 \mathrm{~h}$ and filtered through a $0.45 \mu \mathrm{m}$ filter. The virus was concentrated by ultracentrifugation at $36000 \mathrm{rpm}$ for $4 \mathrm{~h}$ pelleting through a $20 \%$ sucrose cushion or by Centrifugal Filters $(100 \mathrm{Kd})$. Detection of virus capsid release into the cell cytoplasm was performed using LiveBLAzer FRET-B/G Loading Kit with CCF4-AM (Invitrogen) according to manufacturer instructions. Briefly, HeLa or SupT1 cells were seeded on 6-well plates 1 day before the assay. After preincubation with each drug for $1 \mathrm{~h}$, cells were infected with EBOV-GP/HIV-luc or VSV-GP/HIV-luc for $2 \mathrm{~h}$ at $37^{\circ} \mathrm{C}$ in the presence of the drug. After loading CCF4-AM substrate, cells were fixed in $4 \%$ paraformaldehyde and Amcyan or Pacific Blue fluorescence signals were detected by FACS.

\section{Time-of-addition experiment}

Berbamine hydrochloride $(1 \mu \mathrm{M})$, Tetrandrine $(1 \mu \mathrm{M})$ and Efavirenz $(5 \mathrm{nM})$ were added to the 293T cell culture medium before infection $(-1 \mathrm{~h})$, during infection $(0 \mathrm{~h})$, and 1, 4, 5, 6, 8, 18, and 24 hpi. Viral replication was determined at $48 \mathrm{hpi}$ as described above. Cell culture treated with DMSO or TET was used as 
negative and positive control, respectively. The HIV-1 reverse transcriptase inhibitor EFV was used to confirm the rationality of our assay system.

\section{Virus binding experiment}

For binding tests, $4 \times 10^{5}$ HEK293T cells were seeded in 6-well plates overnight and infected with EBOV-GP/HIV-luc for $1 \mathrm{~h}$ at $4^{\circ} \mathrm{C}$ in the presence of Tetrandrine $(5 \mu \mathrm{M})$, Berbamine hydrochloride $(5 \mu \mathrm{M})$ and Heparin $(5 \mu \mathrm{g} / \mathrm{mL})$, followed by washing with PBS, and virus absorption on the cell surface was quantified through the measurement of viral genomic RNA by qRT-PCR.

\section{In vitro protease cleavage of EBOV-GP/HIV-luc}

Concentrated EBOV-GP/HIV-luc was stocked in HEPES-MES buffer (40 mM HEPES, 40 mM morpholinepropanesulfonic acid, $50 \mathrm{mM} \mathrm{NaCl}, 0.5 \mathrm{mM} \mathrm{CaCl} 2, \mathrm{pH} 7.5$ ), the GP protein was cleaved by 0.5 $\mathrm{mg} / \mathrm{mLthermolysin} \mathrm{(Promega,} \mathrm{Madison,} \mathrm{WI)} \mathrm{at} 37^{\circ} \mathrm{C}$ for $1 \mathrm{~h}$. The reaction was stopped by $0.5 \mathrm{mM}$ EDTA and protease inhibitors. The cleavage efficiencies were confirmed by immunoblotting using anti-Flag antibody. Then HEK293T cells were incubated with the cleaved or uncleaved EBOV-GP/HIV-luc in the presence of the drug for $48 \mathrm{~h}$, the luciferase activities were detected to evaluate the antiviral efficiencies of drugs.

\section{In situ PLA}

In situ PLA was performed using kits from Sigma Aldrich: Duolink ${ }^{\circledR}$ PLA Detection Reagents (DU092101) according to the manufacturer. Briefly, $4 \%$ paraformaldehyde-fixed samples were incubated with blocking solution to saturate nonspecific binding and subsequently with primary antibodies at $37^{\circ} \mathrm{C}$ for $1 \mathrm{~h}$. Thereafter, slides were incubated for $1 \mathrm{~h}$ at $37^{\circ} \mathrm{C}$ with Duolink ${ }^{\circledR}$ PLA probes. Ligation solution was added for $30 \mathrm{~min}$ at $37^{\circ} \mathrm{C}$. Ligation solution was removed with wash buffer $\mathrm{A}$, and amplification solution was added for $100 \mathrm{~min}$ at $37^{\circ} \mathrm{C}$ and removed with wash buffer B. Finally, Duolink in situ mounting medium with DAPI was added. Fluorescence was monitored with a confocal microscope with a 100x oil objective lens.

\section{Co-IP for Ebola GPcl and NPC1}

HEK293T cells were transfected with plasmid expressing NPC1-C-Flag using Lipo2000 in $10 \mathrm{~cm}$ dishes for $48 \mathrm{~h}$, cells were lysed by NP40 and debris was removed through centrifugation at $12000 \mathrm{rpm}$ for $10 \mathrm{~min}$.

394 Tetrandrine $(10 \mu \mathrm{M})$, Berbamine hydrochloride $(10 \mu \mathrm{M})$ were pre-incubated with GPcl-His $(1 \mu \mathrm{g})$ for $1 \mathrm{~h}$ 395 before mixing with cell lysate. Anti-His antibody coated beads were used to pull down GPcl-His and the 396 bound NPC1-C-Flag was detected through WB.

\section{Analysis of intracellular cholesterol} Hela cells were pretreated with Tetrandrine $(5 \mu \mathrm{M})$, Berbamine hydrochloride $(5 \mu \mathrm{M})$, U18666A (5 $\mu \mathrm{M})$ for $24 \mathrm{~h}$, and then cells were collected and fixed with paraformaldehyde (4\%) for 30 min on ice. After three PBS

400 washes, cells were incubated with $50 \mu \mathrm{g} / \mathrm{mL}$ filipinIII complex in the dark for $1 \mathrm{~h}$ at RT. After three PBS washes, cells were detected by FACS in the DAPI channel. 
Bio-layer Interferometry (BLI) binding assay

403

404

405

406

407

408

409

410

411

412

413

414

415

416

417

418

419

420

421

422

423

424

425

426

427

428

429

430

431

432

433

434

435

436

Octet RED (ForteBio, Inc., CA, USA) was used to measure binding affinity and kinetic profile. The purified GPcl was biotinylated by EZ-Link NHS-LC-LC-Biotin (Thermo Scientific). The biotinylated GPcl (50 $\mu \mathrm{g} / \mathrm{ml}$ ) was then captured via super streptavidin (SSA) biosensors (120 sec, at $30^{\circ} \mathrm{C}$, with $1000 \mathrm{rpm}$ ). A duplicate set of sensors were incubated in assay buffer $(0.002 \%$ Tween-20, PBS, $\mathrm{pH}=7.4)$ without protein for use as a background binding control. Both the ligand and reference biosensors were quenched with $5 \mu \mathrm{g} / \mathrm{ml}$ biotin for $1 \mathrm{~min}$. For KD determinations, the binding of a dilution series of compounds was detected for $60 \mathrm{sec}$ association (kon, 1/Ms) followed by $60 \mathrm{sec}$ dissociation (kdis, 1/s) in parallel to the ligand biosensors and reference biosensors. Besides, blank binding cycles using buffer only were used to correct the baseline shift during the analysis. After measurements were complete, a double reference subtraction method was processed to subtract the effect of baseline drift and nonspecific binding. KD acquired from fitting into 1:1 binding model by global fitting of multiple kinetic traces and analyzed by Data Analysis 9.0 software.

\section{Computational methods}

Using Auto-Dock Vina package(33), structure based virtual screening was carried out over 4114 compounds in the focused database. Based on the crystal structure of GPcl in complex with the receptor, we first generated the docking parameter files using AutoDockTools-1.5.6. The grid center was located at the receptor-binding region on the head of GPcl and was designated at dimensions ( $x, y$, and $z$ ): $34.428 \AA$, $-17.200 \AA$, and $-317.00 \AA$. The grid size was set to $24 \AA, 28 \AA$, and $28 \AA$ on $X, Y$ and $Z$ coordinates, respectively. Then we used Open Babel software to create the ligand parameter files for docking(34). Each compound in the library was assigned hydrogen at pH 7.4 and converted to PDBQT format. Finally, all these compounds were docked to $\mathrm{GPCl}$ and ranked by their calculated binding free energies $\left(\Delta \mathrm{G}_{\mathrm{ADV}}\right)$. The molecular dynamic simulation and MM-GBSA calculation were performed as previously described(24, 35).

\section{Animal ethics statement}

Animal experiments were performed in the containment level 4 laboratories at CSCHAH. All experimental protocols were reviewed and approved by the institutional Animal Care Committee in accordance with guidelines from the Canadian Council on Animal Care. All staff working on animal experiments completed education and training programs according to the standard protocols appropriate for this level of biosafety.

\section{Efficay of berbamine hydrochloride in EBOV-infected mice}

Six- to eight-week-old female BALB/c mice (Charles River) were inoculated intraperitoneally with 1,000x the $50 \%$ lethal dose (LD50) of MA-EBOV. Mice were treated with $100 \mathrm{mg} / \mathrm{kg}$ of body weight of berbamine hydrochloride via oral administration beginning on either day -1 or day +1 post-infection and continuing daily up to day 6 post-infection. Animals in the control group were treated with PBS following the same schedule. Mice were observed daily for weight change and clinical signs.

\section{Statistical analysis}


438 Statistical comparisons of the Kaplan-Meier survival curves (Fig. 5b, d) were performed using the Log-rank

440

441

442 
2. S. T. Jacob et al., Ebola virus disease. Nature reviews Disease primers 6, 1-31 (2020).

V. E. Volchkov et al., Recovery of infectious Ebola virus from complementary DNA: RNA editing

450 of the GP gene and viral cytotoxicity. Science 291, 1965-1969 (2001).

6. B. Manicassamy, J. Wang, H. Jiang, L. Rong, Comprehensive analysis of ebola virus GP1 in viral

15. E. Picazo, F. Giordanetto, Small molecule inhibitors of ebola virus infection. Drug Discov Today

16. M. A. Trad et al., Ebola virus disease: An update on current prevention and management

17. Yasuteru Sakurai et al., Two-pore channels control Ebola virus host cell entry and are drug targets for disease treatment. Science 347, 995-998 (2015).

18. H. Cheng et al., Inhibition of Ebola and Marburg Virus Entry by G Protein-Coupled Receptor Antagonists. J Viro/89, 9932-9938 (2015).

19. A. Basu et al., Identification of a small-molecule entry inhibitor for filoviruses. $J$ Viro/ 85 , 3106-3119 (2011).

20. X. Zhang et al., Characterization of the inhibitory effect of an extract of Prunella vulgaris on 
Ebola virus glycoprotein (GP)-mediated virus entry and infection. Antiviral Res 127, 20-31 (2016).

21. J. Ren, Y. Zhao, E. E. Fry, D. I. Stuart, Target Identification and Mode of Action of Four Chemically Divergent Drugs against Ebolavirus Infection. J Med Chem 61, 724-733 (2018).

22. Y. Zhao et al., Toremifene interacts with and destabilizes the Ebola virus glycoprotein. Nature 535, 169-172 (2016).

23. M. Côté et al., Small molecule inhibitors reveal Niemann-Pick C1 is essential for Ebola virus infection. Nature 477, 344-348 (2011).

24. Q. Li et al., Novel cyclo-peptides inhibit Ebola pseudotyped virus entry by targeting primed GP protein. Antiviral Res 155, 1-11 (2018).

25. X. Zhang et al., Treatment of Chemotherapy Related Leukocytopenia by Oral Administration of Multiple Leucogenic Drugs Combined with G-CSF: an Experimental Study. Chin J Integr Med 35, 860-865 (2015).

26. A. O'Hearn et al., Role of EXT1 and Glycosaminoglycans in the Early Stage of Filovirus Entry. J Viro/89, 5441-5449 (2015).

27. M. F. Saeed, A. A. Kolokoltsov, T. Albrecht, R. A. Davey, Cellular entry of ebola virus involves uptake by a macropinocytosis-like mechanism and subsequent trafficking through early and late endosomes. PLoS pathogens 6, e1001110 (2010).

28. A. Nanbo et al., Ebolavirus is internalized into host cells via macropinocytosis in a viral glycoprotein-dependent manner. PLoS pathogens 6, e1001121 (2010).

29. M. Cavrois, C. De Noronha, W. C. Greene, A sensitive and specific enzyme-based assay detecting HIV-1 virion fusion in primary T lymphocytes. Nature biotechnology 20, 1151-1154 (2002).

30. T. Hashiguchi et al., Structural basis for Marburg virus neutralization by a cross-reactive human antibody. Cel/160, 904-912 (2015).

31. H. Wang et al., Ebola Viral Glycoprotein Bound to Its Endosomal Receptor Niemann-Pick C1. Cel/ 164, 258-268 (2016).

32. M. Bray, K. Davis, T. Geisbert, C. Schmaljohn, J. Huggins, A mouse model for evaluation of prophylaxis and therapy of Ebola hemorrhagic fever. The Journal of infectious diseases $\mathbf{1 7 8 ,}$ 651-661 (1998).

33. O. Trott, A. J. Olson, AutoDock Vina: improving the speed and accuracy of docking with a new scoring function, efficient optimization, and multithreading. J Comput Chem 31, 455-461 (2010).

34. N. M. O'Boyle et al., Open Babel: An open chemical toolbox. J Cheminform 3, 33 (2011).

35. T. Hou, J. Wang, Y. Li, W. Wang, Assessing the performance of the MM/PBSA and MM/GBSA methods. 1. The accuracy of binding free energy calculations based on molecular dynamics simulations. J. Chem. Inf. Model. 51, 69-82 (2010). 


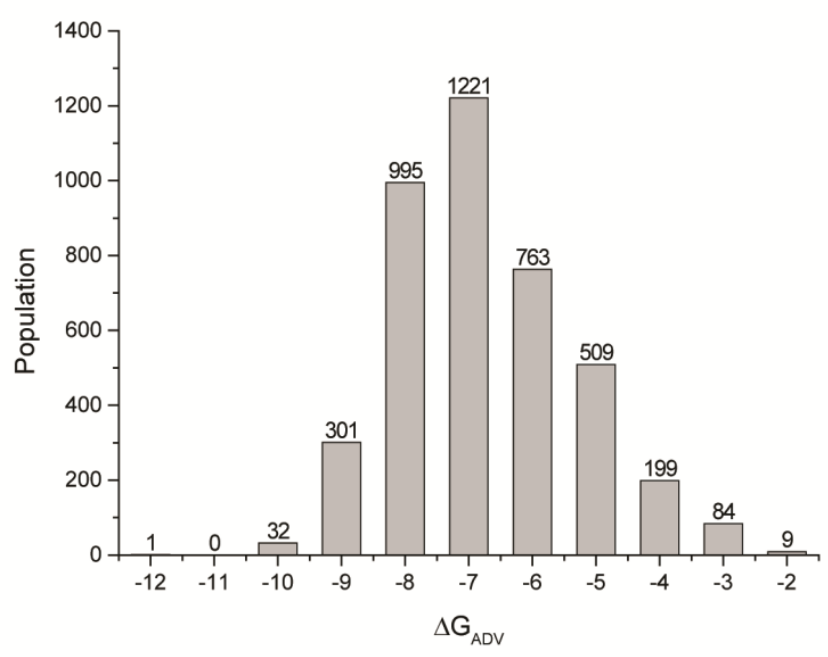

Extended Data Fig. S1. Virtual screening of small molecule compound library against GPcl. Bars represent numbers of compounds with predicted free energies of binding in the indicated $1 \mathrm{kcal}$ mol-1 bins.

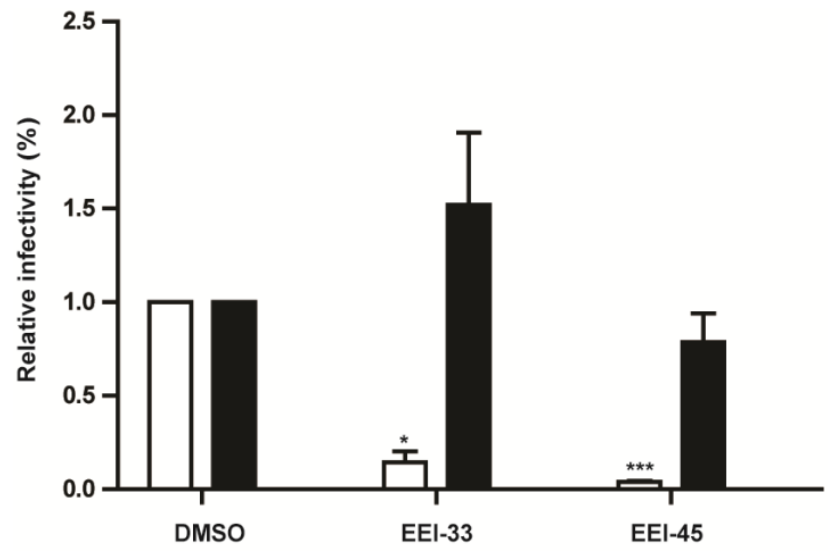

Extended Data Fig. S2. Inhibition of EBOVpp and VSVpp infection by EEI-33 and EEI-45. HEK293T cells were infected with viruses EBOVpp (white bar) or VSVpp (black bar) in the presence of EEI-33 or EEI-45 at the final concentration of 10 $\mu \mathrm{M}$. Data shown are the means \pm s.d. $n=3$. Value of the control is arbitrarily set as 1 . 


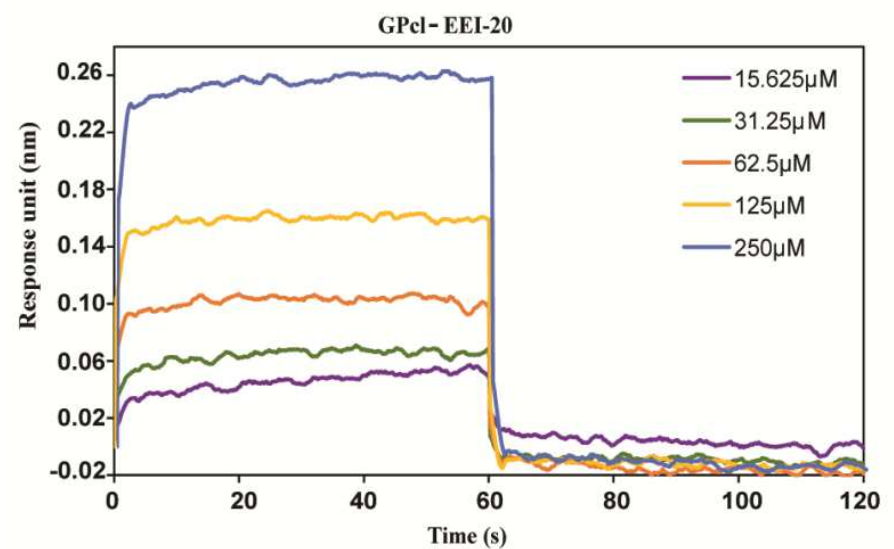

Extended Data Fig. S3. EEI-20 interacts with Ebola GPcl. Representative association and dissociation sensorgrams of EEI-20 binding to EBOV-GPCl. Values of KD, KD Error, kon, kdis, and R2 are shown.

a

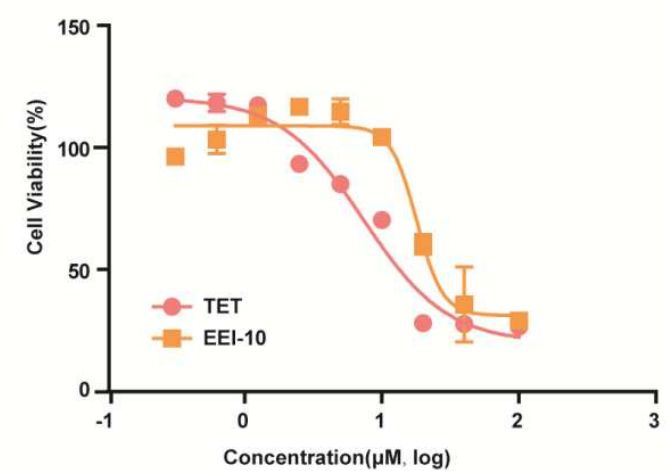

b

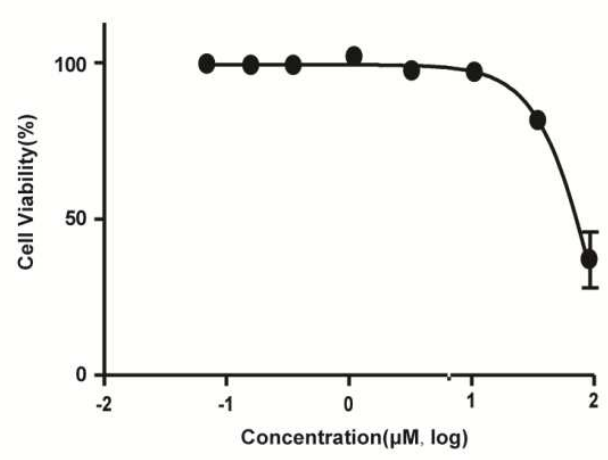

547 Extended Data Fig. S4. Cytotoxic effects of EEI-10 and TET on HEK293T cells. HEK293T (a) or Vero E6 (b) cells were 548 incubated with EEI-10 or TET at different concentrations. The cell viability was analyzed by CCK8 assay. Data shown are 549 the means \pm s.d. $n=3$. 

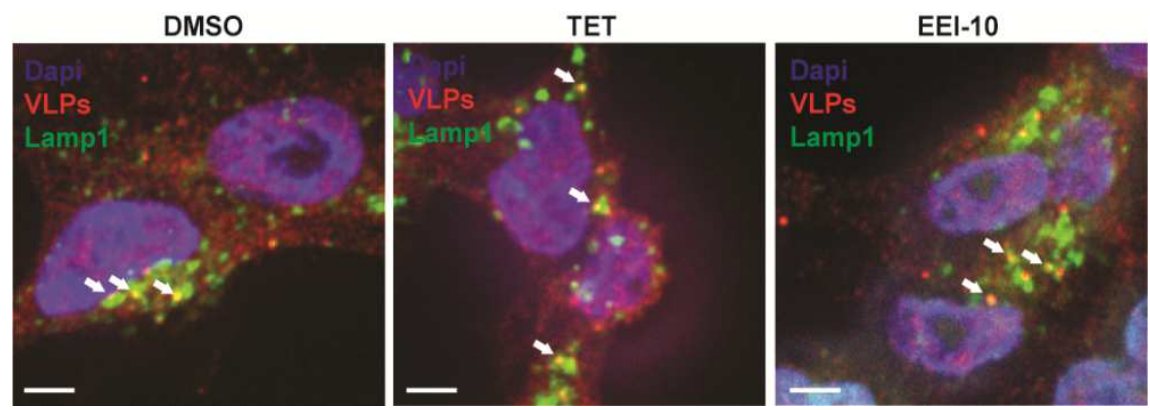

Extended Data Fig. S5. EEI-10 does not affect the transport of Ebola virus to late endosomes. HEK293T cells were incubated with Ebola VLPs for 4 hours in the presence of EEI-10 or TET. Colocalization of Ebola VLPs (in red) with Lamp1 (in green) was determined by confocal microscopy. Arrowheads indicate the colocalized

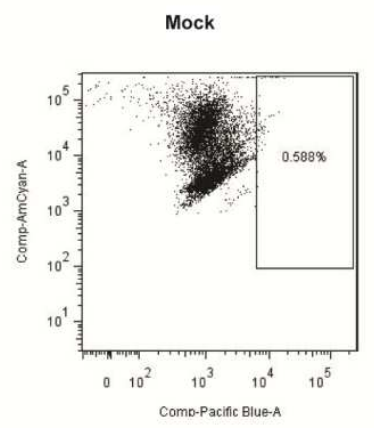

b

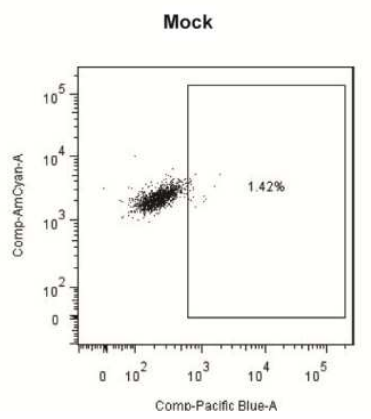

DMSO

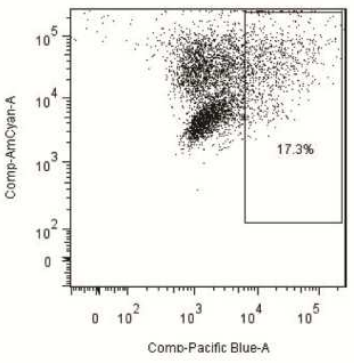

DMSO

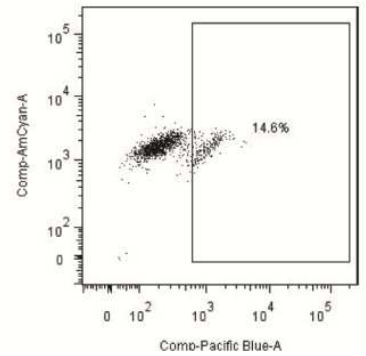

TET

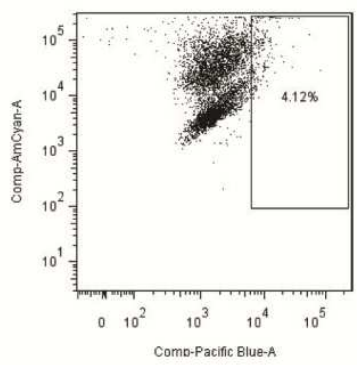

TET

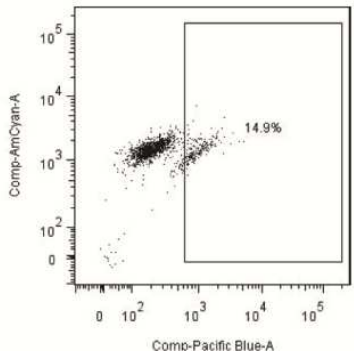

EEI-10

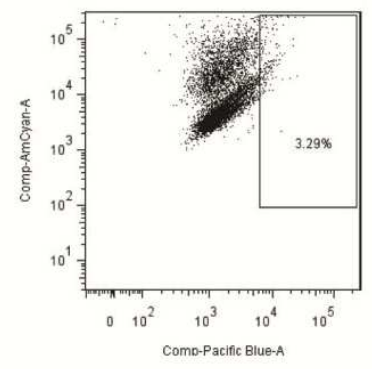

EEI-10

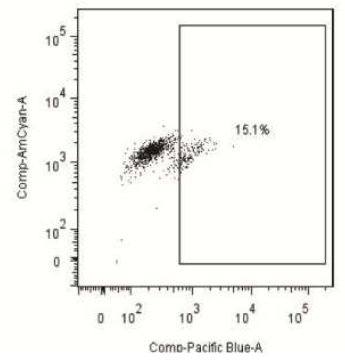

Extended Data Fig. S6. EEI-10 inhibits EBOV GP-mediated membrane fusion. a, HeLa cells were infected with EBOVpp loaded with BlaM-Vpr in the presence of EEI-10. Release of virus capsid was determined by measuring cytoplasmic B-lactamase activity using the CCF4-AM Kit. Results shown are the representative FACS data of three independent experiments. b, BlaM-Vpr assay was performed to measure the effect of EEI-10 on VSVpp capsid release by infecting the HEK293T cells. 
a
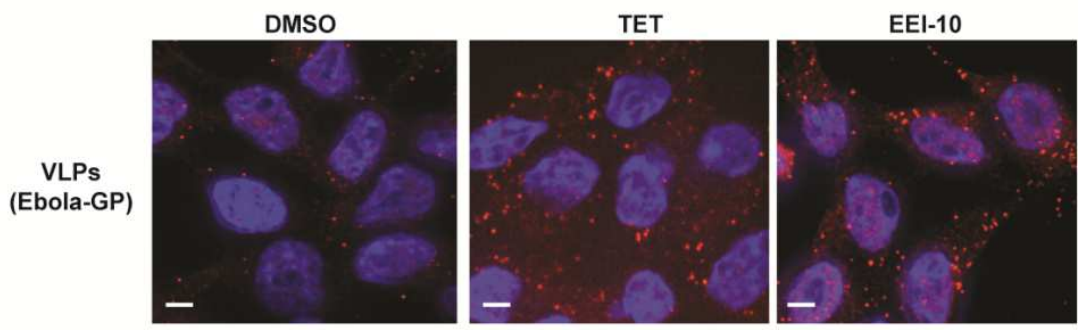

b
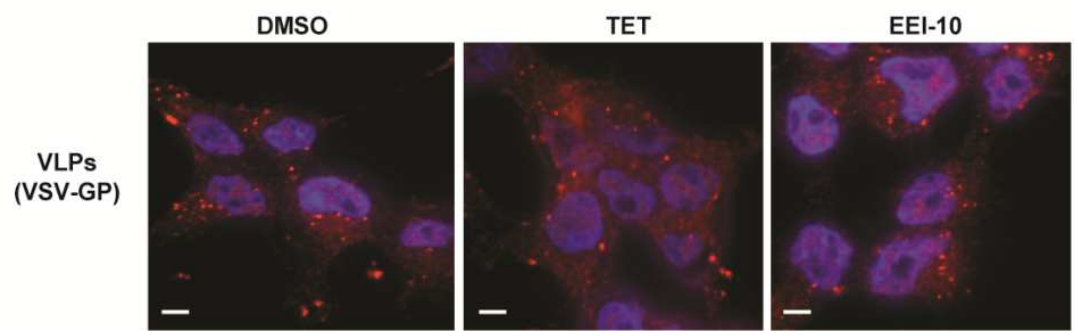

Extended Data Fig. S7. EEI-10 causes accumulation of Ebola virus in the cytoplasm. HEK293T cells were incubated with Ebola-GP/HIV-Iuc (a) or VSV-GP/HIV-luc (b) under the treatment of EEI-10 or TET. Representative confocal images of virus particles (in red) from three independent infection experiments are shown. Scale bars, $5 \mu \mathrm{m}$.
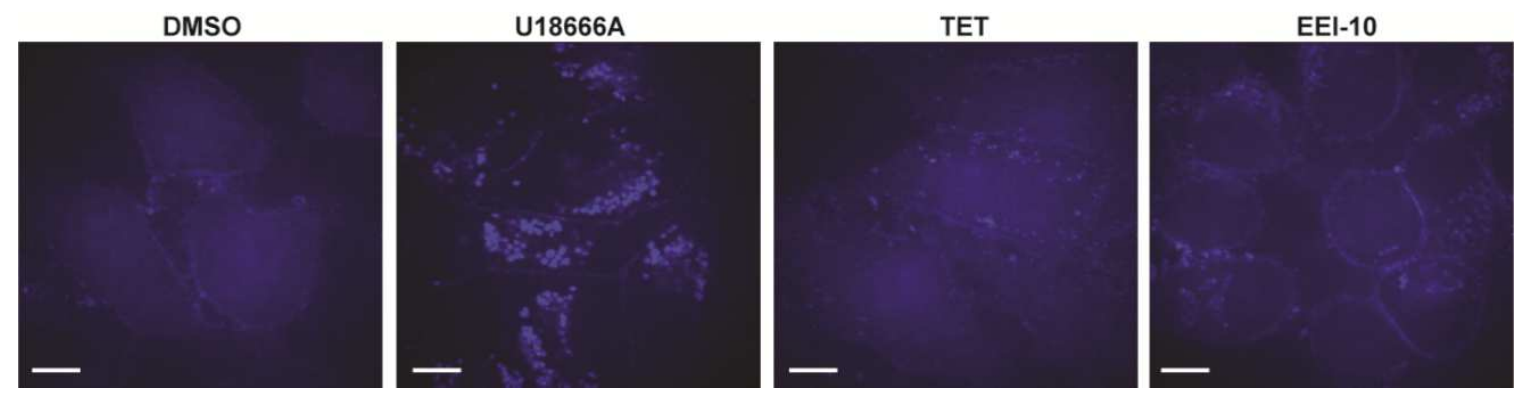

579 Extended Data Fig. S8. EEI-10 does not affect the cholesterol transport. HEK293T cells were treated with EEI-10 ( $5 \mu \mathrm{M})$,

$580 \mathrm{TET}(5 \mu \mathrm{M})$ or U18666A $(5 \mu \mathrm{M})$, then stained with filipinIl (in blue). Representative confocal images are shown. Scale 581 bars, $5 \mu \mathrm{m}$. 


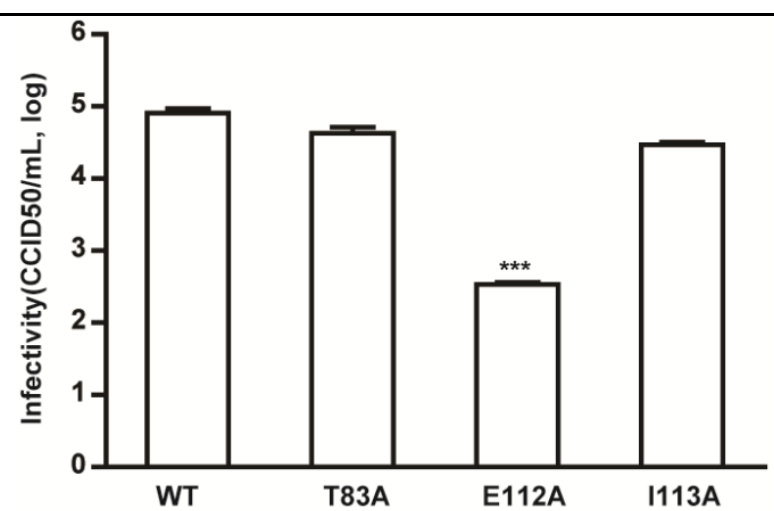

588 Extended Data Fig. S9. Infectivity of EBOV GP mutants. HEK293T cells were infected with EBOVpp with Ebola GP (WT、

589 T83A、E112A $、$ I113A) at different dilutions for 48h, and viral infectivity were determined by measurement of luciferase 590 activities, followed by calculation of viral titers. Data are mean \pm s.d. $n=3$.

591

592 Extended Data Table S1. Information of the selected 46 compounds, including the predicted binding free 593 energies $\left(\Delta G_{A D V}\right)$, TargetMol ID, CAS number, formula, and molecule weight (MolWt).

\begin{tabular}{|c|c|c|c|c|c|}
\hline No. & ID & $\begin{array}{c}\Delta \mathbf{G}_{\mathrm{ADV}} \\
(\mathrm{Kcal} / \mathrm{mol})\end{array}$ & CAS & Formula & MolWt \\
\hline 1 & T1928 & -10.2 & 875446-37-0 & $\mathrm{C} 30 \mathrm{H} 25 \mathrm{~F} 10 \mathrm{NO} 3$ & 637.51 \\
\hline 2 & T0047 & -8.7 & $357-57-3$ & $\mathrm{C} 23 \mathrm{H} 26 \mathrm{~N} 2 \mathrm{O} 4$ & 394.47 \\
\hline 3 & T0722 & -9.2 & $77-52-1$ & $\mathrm{C} 3 \mathrm{OH} 48 \mathrm{O} 3$ & 456.71 \\
\hline 4 & T0878 & -9.4 & $520-27-4$ & $\mathrm{C} 28 \mathrm{H} 32 \mathrm{O} 15$ & 608.55 \\
\hline 5 & T1842 & -10.4 & $1435488-37-1$ & $\mathrm{C} 36 \mathrm{H} 46 \mathrm{~N} 8 \mathrm{O} 3$ & 638.81 \\
\hline 6 & T2348 & -10.2 & $1227911-45-6$ & $\mathrm{C} 25 \mathrm{H} 34 \mathrm{~N} 8 \mathrm{O}$ & 462.59 \\
\hline 7 & T2585 & -8.9 & 843663-66-1 & $\mathrm{C} 32 \mathrm{H} 31 \mathrm{BrN} 2 \mathrm{O} 2$ & 555.5 \\
\hline 8 & T2768 & -8.7 & 20736-09-8 & $\mathrm{C} 42 \mathrm{H} 68 \mathrm{O} 13$ & 780.99 \\
\hline 9 & T0049 & -8.9 & $37091-65-9$ & $\mathrm{C} 2 \mathrm{OH} 22 \mathrm{~N} 5 \mathrm{NaO} 6 \mathrm{~S}$ & 483.47 \\
\hline 10 & T2920 & -10.9 & $6078-17-7$ & C37H41CIN2O6 & 645.19 \\
\hline 11 & T0132 & -9 & $33419-42-0$ & $\mathrm{C} 29 \mathrm{H} 32 \mathrm{O} 13$ & 588.56 \\
\hline 12 & T3122 & -9.8 & $436-77-1$ & C37H4ON2O6 & 608.74 \\
\hline 13 & T1304 & -9.4 & $64-72-2$ & $\mathrm{C} 22 \mathrm{H} 23 \mathrm{CIN} 2 \mathrm{O} 8 . \mathrm{HCl}$ & 515.35 \\
\hline 14 & T1138 & -9.8 & $345909-26-4$ & $\mathrm{C} 26 \mathrm{H} 46 \mathrm{NNaO} 8 \mathrm{~S}$ & 555.7 \\
\hline
\end{tabular}




\begin{tabular}{|c|c|c|c|c|c|}
\hline 15 & T0795 & -8.7 & $153-18-4$ & $\mathrm{C} 27 \mathrm{H} 30016$ & 610.52 \\
\hline 16 & T2728 & -9.7 & $1180-71-8$ & $\mathrm{C} 26 \mathrm{H} 3008$ & 470.52 \\
\hline 17 & T2830 & -9.9 & $472-15-1$ & $\mathrm{C} 30 \mathrm{H} 48 \mathrm{O} 3$ & 456.71 \\
\hline 18 & T0595 & -8.6 & $10236-47-2$ & $\mathrm{C} 27 \mathrm{H} 32 \mathrm{O} 14$ & 580.54 \\
\hline 19 & T2755 & -8.7 & $17306-46-6$ & $\mathrm{C} 27 \mathrm{H} 30 \mathrm{O} 14$ & 578.52 \\
\hline 20 & T0125 & -8.8 & 56390-09-1 & C27H30CINO11 & 579.98 \\
\hline 21 & T0171 & -10.8 & $68-23-5$ & $\mathrm{C} 2 \mathrm{OH} 26 \mathrm{O} 2$ & 298.42 \\
\hline 22 & T1521 & -8.9 & $64439-81-2$ & $\mathrm{C} 2 \mathrm{OH} 16 \mathrm{~N} 2 \mathrm{O} 5$ & 364.36 \\
\hline 23 & T6025 & -9.6 & $850876-88-9$ & C35H46FN5O9S & 731.84 \\
\hline 24 & $\mathrm{~T} 1213$ & -8.9 & $59703-84-3$ & $\mathrm{C} 23 \mathrm{H} 26 \mathrm{~N} 5 \mathrm{NaO} 7 \mathrm{~S}$ & 539.54 \\
\hline 25 & T2831 & -9.2 & $475-31-0$ & $\mathrm{C} 26 \mathrm{H} 43 \mathrm{NO} 6$ & 465.63 \\
\hline 26 & T1541 & -10.3 & $67392-87-4$ & $\mathrm{C} 24 \mathrm{H} 30 \mathrm{O}$ & 366.49 \\
\hline 27 & T1259 & -9.2 & $22204-24-6$ & C34H30N2O6S & 594.69 \\
\hline 28 & T1571 & -9.4 & $50-27-1$ & $\mathrm{C} 18 \mathrm{H} 24 \mathrm{O} 3$ & 288.39 \\
\hline 29 & T0129 & -10.2 & $5578-73-4$ & $\mathrm{C} 2 \mathrm{OH} 14 \mathrm{CINO} 4$ & 367.79 \\
\hline 30 & T1832 & -9.7 & $878419-78-4$ & $\mathrm{C} 14 \mathrm{H} 10 \mathrm{~F} 3 \mathrm{~N} 5 \mathrm{O} 2$ & 337.26 \\
\hline 31 & T1157 & -10.8 & $113507-06-5$ & C37H53NO8 & 639.84 \\
\hline 32 & T2866 & -10.5 & $510-30-5$ & $\mathrm{C} 30 \mathrm{H} 48 \mathrm{O} 4$ & 472.71 \\
\hline 33 & T1775 & -9.4 & $1346572-63-1$ & $\mathrm{C} 31 \mathrm{H} 38 \mathrm{~N} 6 \mathrm{O} 2$ & 526.69 \\
\hline 34 & T2733 & -10.2 & $126-19-2$ & $\mathrm{C} 27 \mathrm{H} 44 \mathrm{O} 3$ & 416.65 \\
\hline 35 & T2907 & -9.2 & $568-73-0$ & $\mathrm{C} 18 \mathrm{H} 12 \mathrm{O} 3$ & 276.29 \\
\hline 36 & T1743 & -10.1 & $170729-80-3$ & $\mathrm{C} 23 \mathrm{H} 21 \mathrm{~F} 7 \mathrm{~N} 4 \mathrm{O} 3$ & 534.43 \\
\hline 37 & T2239L & -8.8 & 518048-05-0 & $\mathrm{C} 20 \mathrm{H} 21 \mathrm{FN} 6 \mathrm{O} 5$ & 444.42 \\
\hline 38 & T1101 & -9.3 & $13614-98-7$ & C23H28CIN3O7 & 493.94 \\
\hline 39 & T2405 & -10 & $178307-42-1$ & $\mathrm{C} 22 \mathrm{H} 24 \mathrm{ClFN} 4$ & 398.91 \\
\hline 40 & T2239 & -8.8 & $871038-72-1$ & C2OH2OFN6O5.K & 482.51 \\
\hline 41 & T1035 & -9.3 & $520-26-3$ & $\mathrm{C} 28 \mathrm{H} 34 \mathrm{O} 15$ & 610.57 \\
\hline 42 & T0131 & -12.2 & $481-49-2$ & C37H38N2O6 & 606.73 \\
\hline
\end{tabular}




\begin{tabular}{rrrccc}
\hline 43 & T1499 & -10 & $164656-23-9$ & C27H30F6N2O2 & 528.53 \\
44 & T1220 & -8.6 & $1239-45-8$ & C21H2ON3.Br & 394.32 \\
45 & T2100 & -10.5 & $1138549-36-6$ & C27H27N7O2S & 513.61 \\
46 & T2906 & -9.4 & $568-72-9$ & C19H18O3 & 294.35 \\
\hline
\end{tabular}

594

595

596

597

598

599

600 Extended Data Table S2. Calculation of the interaction energy between EEI-10 and amino resides of EBOV-GPcl.

\begin{tabular}{cccccc} 
Residue & van der Waals & Electrostatic & Polar Solvation & Non-Polar Solv. & TOTAL \\
\hline G112 & 0.12 & -3.73 & 1.10 & -0.02 & -2.54 \\
T83 & -3.04 & -0.39 & 1.38 & -0.43 & -2.49 \\
I113 & -1.88 & -0.46 & 0.32 & -0.21 & -2.22 \\
V141 & -1.29 & -0.91 & 0.70 & -0.16 & -1.66 \\
F88 & -1.73 & -0.16 & 0.77 & -0.28 & -1.41 \\
L111 & -1.10 & -0.02 & 0.07 & -0.12 & -1.17 \\
W86 & -1.61 & -0.21 & 1.14 & -0.17 & -0.85 \\
V79 & -0.78 & 0.05 & 0.00 & -0.08 & -0.81 \\
P80 & -1.12 & -0.18 & 0.70 & -0.20 & -0.80 \\
G143 & -0.85 & -0.24 & 0.58 & -0.11 & -0.61 \\
\hline
\end{tabular}

601 aThe total energy ( $\left.\mathrm{kcal} \mathrm{mol}^{-1}\right)$ is the sum of that of van der Waals, Electrostatic, Polar Solvation, and Non-Polar Solvation.

602 The residues are ranked by the values of their total energy. The top 10 residues are shown.

603

604

605

606 


\begin{tabular}{cccccc}
\hline Extended Data Table S3. Calculation of the interaction energy between EEI-10 and amino acid residues of MARV-GP1a. \\
\hline Residue & van der Waals & Electrostatic & Polar Solvation & Non-Polar Solv. & TOTAL \\
\hline I125 & -1.54 & -0.43 & 0.30 & -0.21 & -1.88 \\
$\mathbf{F 7 2}$ & -2.07 & -0.54 & 1.26 & -0.29 & -1.64 \\
$\mathbf{S 6 7}$ & -2.66 & 0.04 & 1.52 & -0.36 & -1.46 \\
$\mathbf{L 6 4}$ & -1.62 & -0.35 & 0.82 & -0.26 & -1.41 \\
$\mathbf{V 9 7}$ & -1.11 & -0.15 & 0.11 & -0.15 & -1.31 \\
$\mathbf{1 9 5}$ & -0.98 & -0.10 & 0.03 & -0.09 & -1.14 \\
$\mathbf{N} 129$ & -1.56 & -1.36 & 2.23 & -0.30 & -0.99 \\
A71 & -0.92 & -0.25 & 0.41 & -0.12 & -0.89 \\
K68 & -1.25 & 0.33 & 0.63 & -0.23 & -0.52 \\
G127 & -0.62 & -0.03 & 0.31 & -0.09 & -0.43 \\
\hline
\end{tabular}

608

aThe total energy ( $\mathrm{kcal} \mathrm{mol}^{-1}$ ) is the sum of that of van der Waals, Electrostatic, Polar Solvation, and Non-Polar Solvation.

610

611

612

613

614

615

616

617

618

619

620

621

622

623 
a

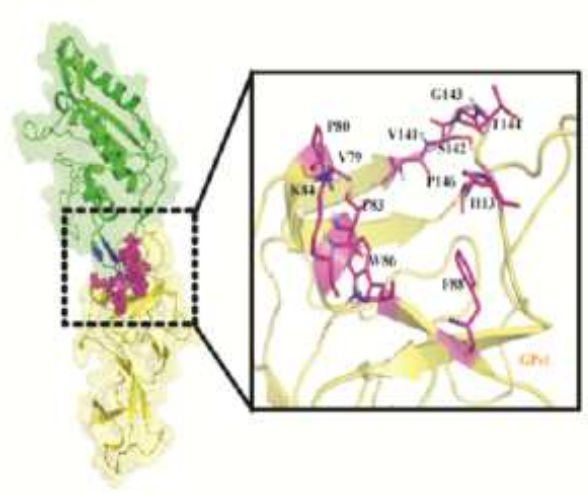

b

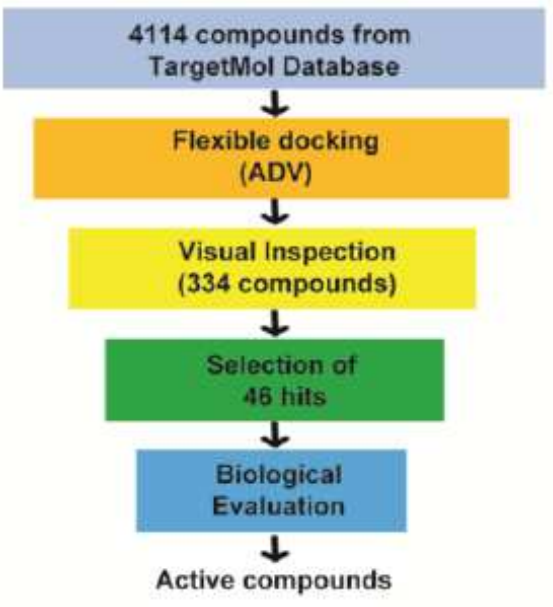

C<smiles>COc1cc2c3cc1Oc1c(OC)cc4c(c1OC)[C@@H](Cc1ccc(O)c(c1)Oc1ccc(cc1)C[C@H]4N(C)CC2)N(C)CC3</smiles>

EEI-10 (Berbamine hydrochloride)

MW,645.19 d

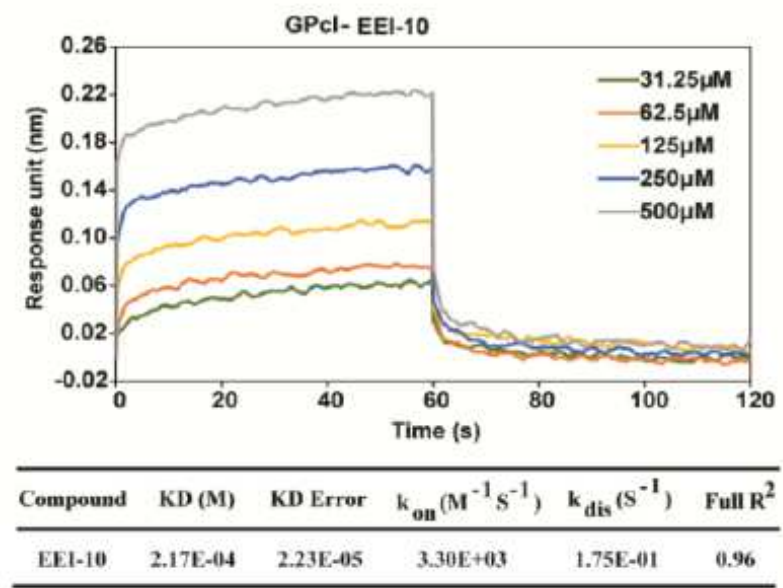

f

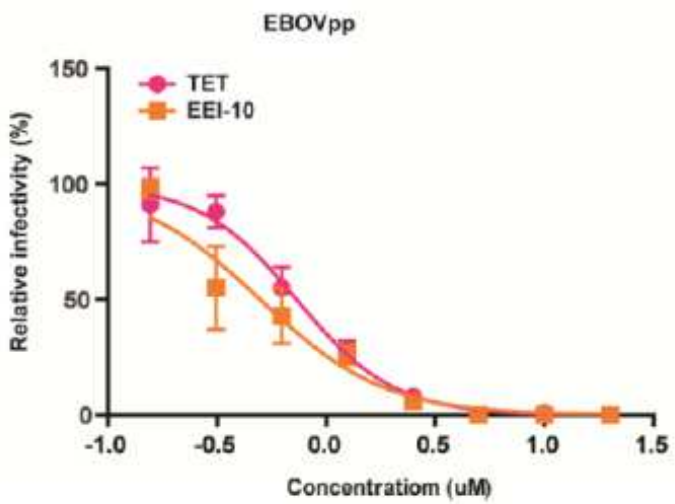

e

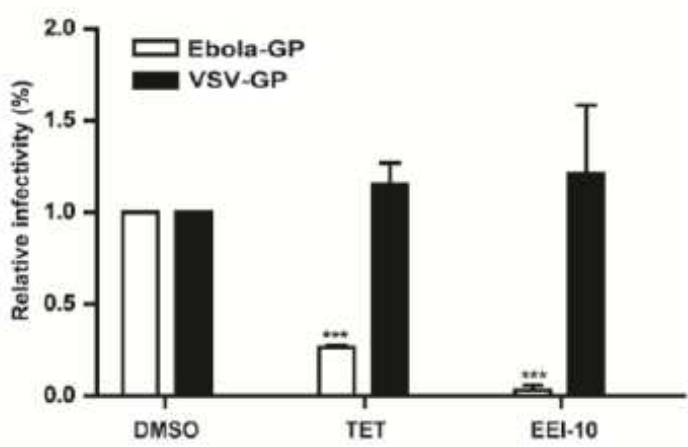

g

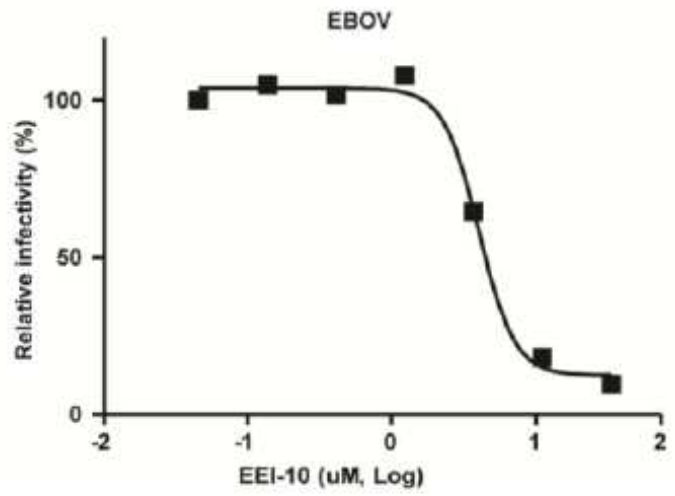

\section{Figure 1}

Identification of EEI-10 as an EBOV entry inhibitor. a, The receptor binding region identified in GPCl (PDB ID 5F1B). Left, the structure of the GPcl (yellow) in complex with NPC1 (green). Right, the receptor binding cavity at the top of GPcl. Highlighted in magenta is residues that constitute the interaction interface and are targeted by the virtual screening. $b$, The virtual screening strategy designed to identify small molecules targeting GPcl. c, Chemical structure of EEl-10, with molecule weight (MW). d, Representative 
association and dissociation sensorgrams of EEI-10 binding to EBOV-GPcl. Values of KD, KD Error, kon, kdis, and R2 are presented. e, HEK293T cells were infected with the EBOVpp in the presence of EEI-10 at the final concentration of $10 \mu \mathrm{M}$. VSVpp was used to control the specific antiviral effect of the tested drugs. Data are normalized to that of the control group (arbitrarily set as 1 ), and shown as means \pm s.d. $n$ = 3. $\mathrm{f}$, Does-depended inhibition of EBOVpp by EEI-10 or TET. $\mathrm{g}$, Inhibition of EBOV infection of Vero E6 cells by EEI-10 of different doses. EC50 and EC90 values were calculated by GraphPad Prism 5.0. Data shown are means \pm s.d. $n=3$. P values were calculated using a two-sided paired t-test.

a

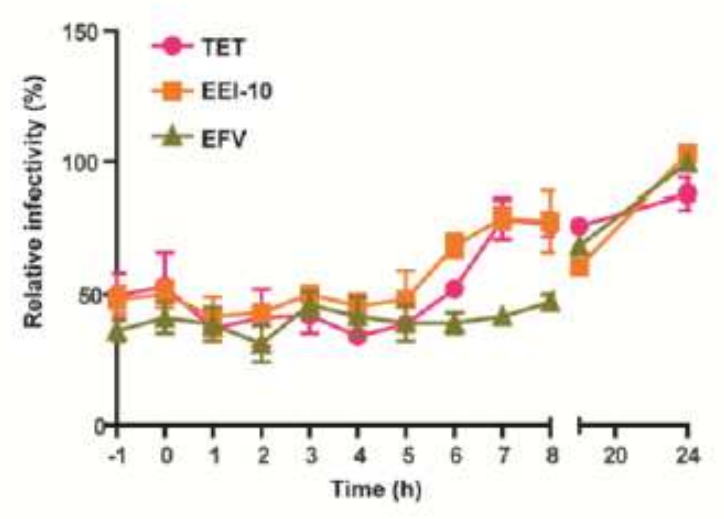

d

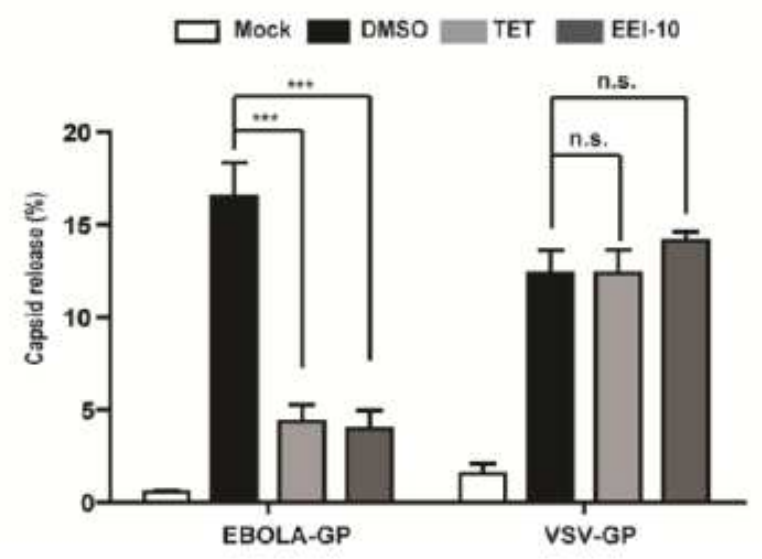

b

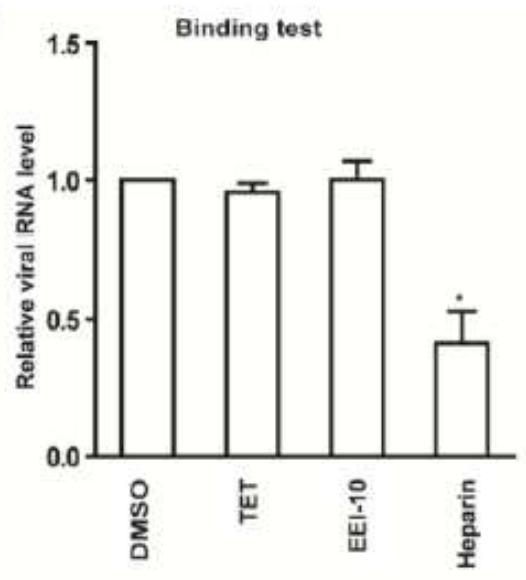

c

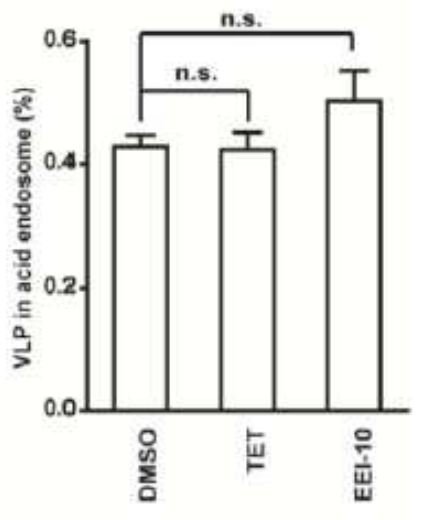

e

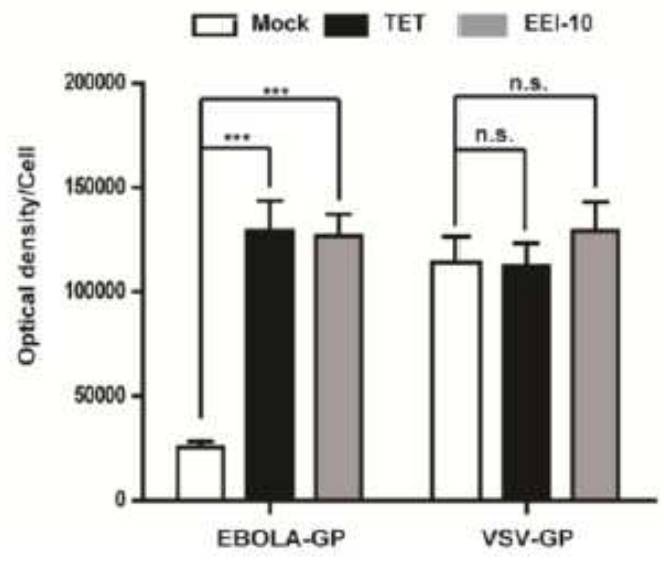

Figure 2

EEI-10 inhibits EBOV-GP dependent virus-cell membrane fusion. a, Time-addition experiment to determine the inhibition mechanism by EEI-10, TET and efavirenz (EFV). Data shown are the means \pm s.d. $n=3 . b$, Binding test to measure the effect of EEl-10 on virus binding to the cell membrane. Heparin was used as a positive control. Viral RNA from virus particles binding to the cell membrane was quantified by qRT-PCR. Data are normalized to that the control group which is arbitrarily set to 1. c, Virus particles (red) in the acidic endosomes were scored by their colocalization with Lamp1 (green) (as for fig. S5). Fluorescence intensity of green and red signals from randomly selected cells $(n \geq 30)$ was quantified by Image-Pro Plus 10 software. d, VLPs (EBOVpp and VSVpp) loaded with BlaM-Vpr were used to measure membrane fusion and virus capsid release into the cytoplasm after EEI-10 and TET treatment by flow cytometry (as 
for fig. S6). The bar graph presents the percentages of virus release into the cytoplasm. Data shown are the means \pm s.d. $n=3$. e, VLPs (EBOVpp and VSVpp) accumulated in the cytoplasm after EEI-10 and TET treatment. The number of VLPs (red signals) in confocal images was determined from randomly selected cells $(n \geq 80)$. The fluorescence intensity was quantified by Image-Pro Plus 10 software. $P$ values were calculated using a two-sided paired t-test.

a
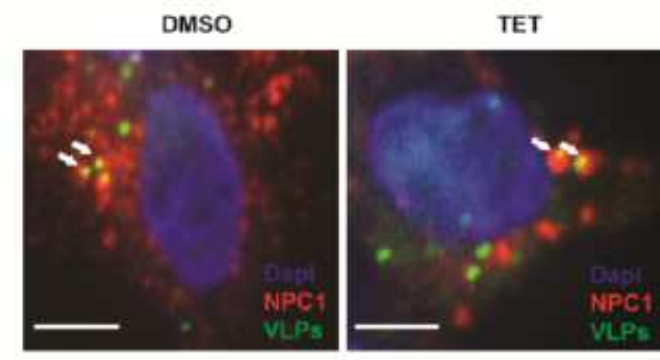

c

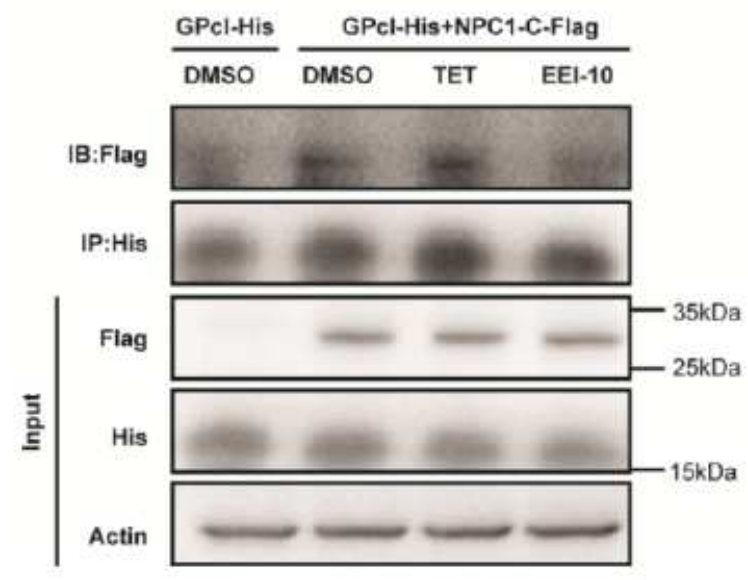

b

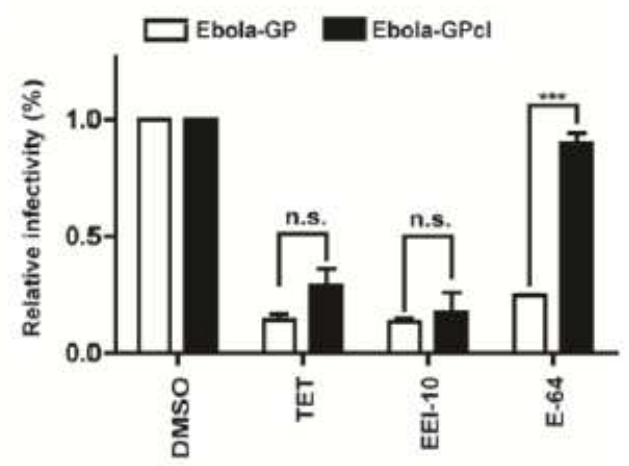

d

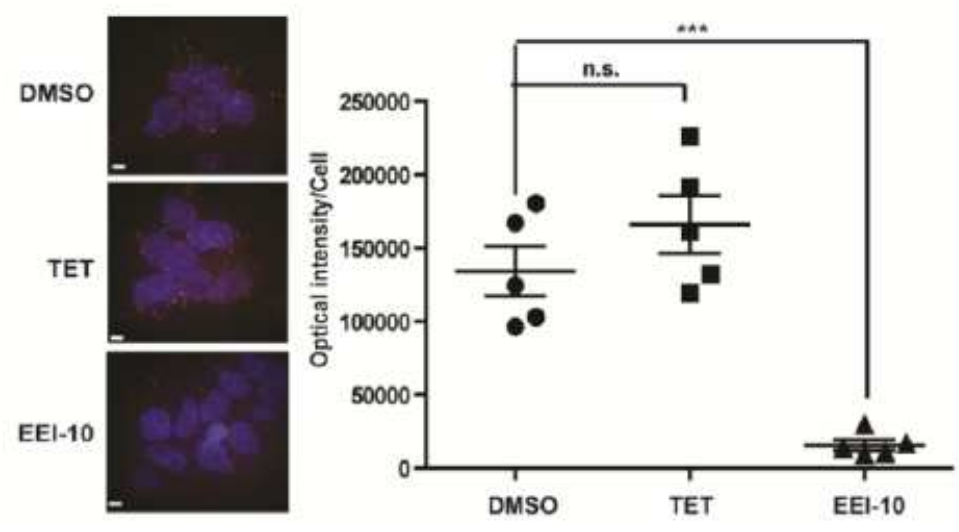

\section{Figure 3}

EEl-10 disrupts the binding of EBOV-GPcl to NPC1. a, Colocalization of Ebola VLPs with NPC1 was determined by incubating VLPs with HEK293 cells for 4 hours. Representative confocal images are shown. Colocalized Ebola VLPs (Green) and NPC1(Red) were indicated by arrowheads. Scale bars, $5 \mu \mathrm{m}$. b, HEK293T cells were exposed to EBOVpp which were treated with protease thermolysin to generate EBVO GPCl, in the presence of EEI-10 or TET. The cysteine protease inhibitor E- 64 was used as a positive control. Luciferase activities were measured and normalized to those of untreated controls (mean $\pm \mathrm{s} . \mathrm{d} . \mathrm{n}$ $=3$ ). Each data set is representative of three independent experiments. c, Interaction between NPC1-CFlag and GPcl-His was determined with Co-IP in the presence of EEI-10 or TET. Data shown are the representative of three independent experiments. $d$, In situ PLA to analyze the interaction between EbolaGP (or VSV-GP) and cellular NPC1 in the presence of the EEI-10 or TET. Representative confocal images are shown. Scale bars, $5 \mu \mathrm{m}$. Mean fluorescence intensity was collected from at least 30 cells and further analyzed for each treatment. 
a

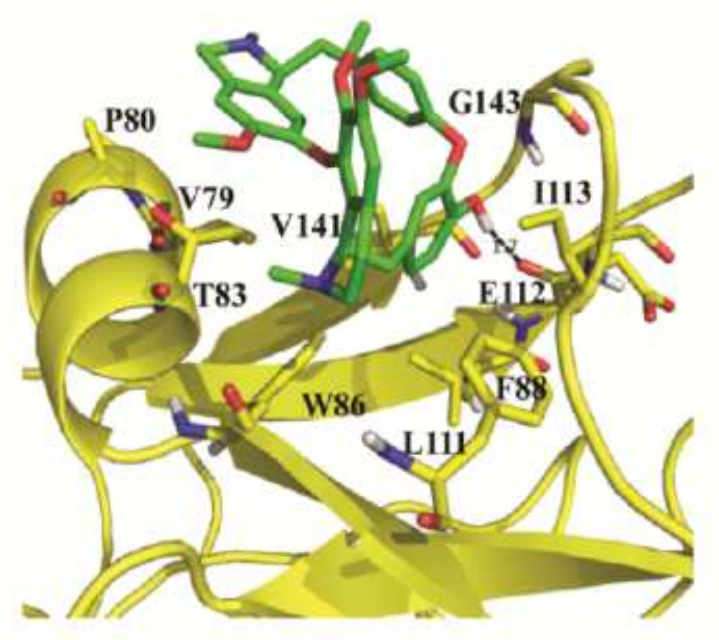

C

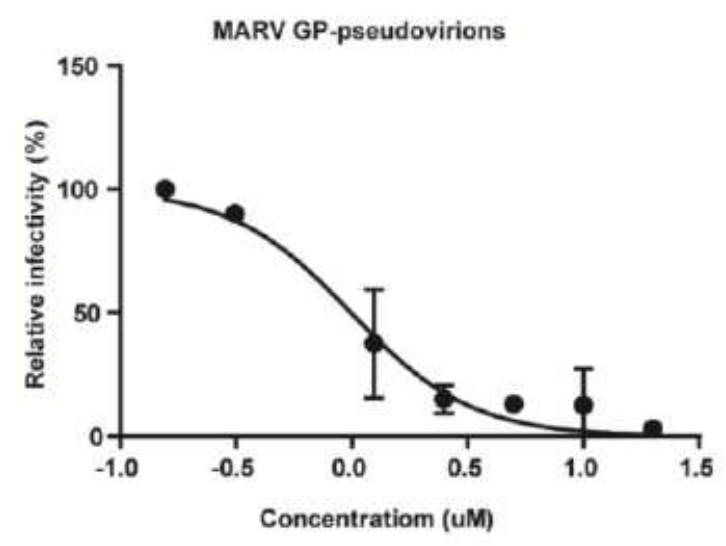

e

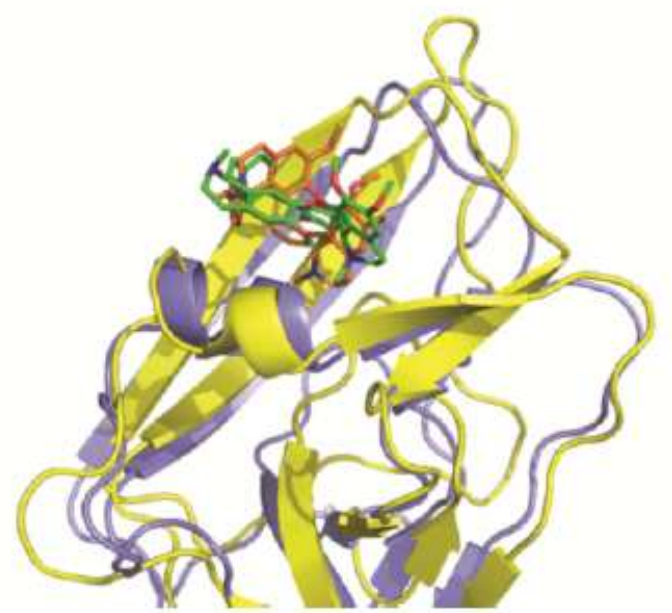

b

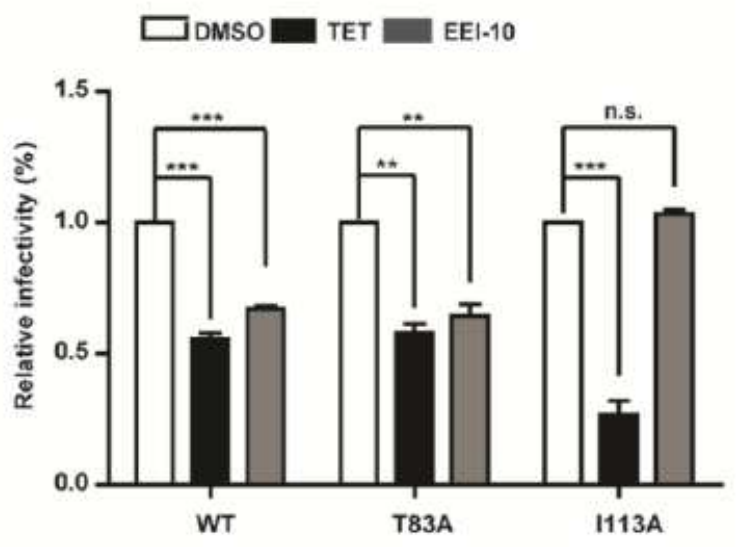

d

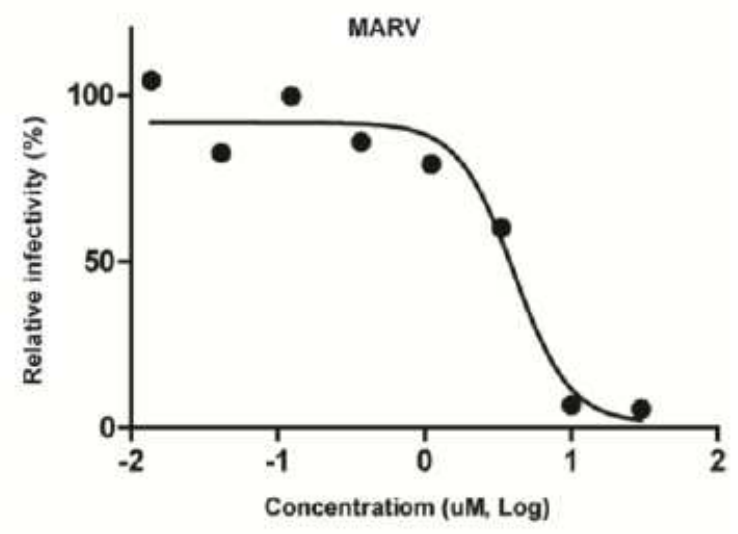

f

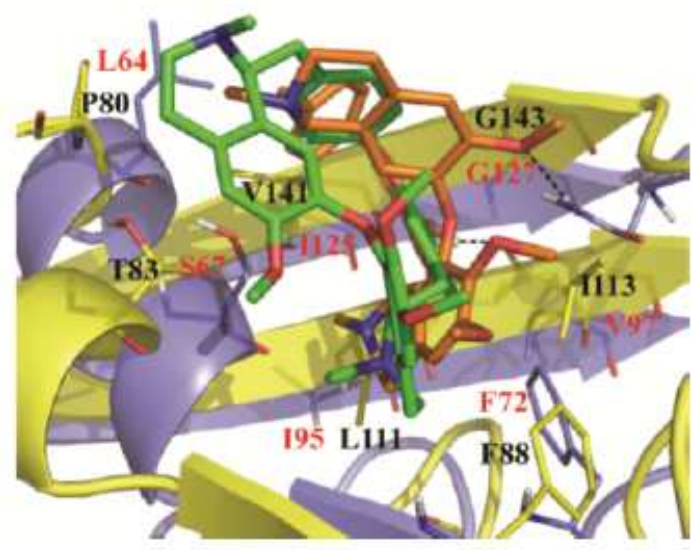

Figure 4

EEI-10 inhibits infection mediated by the GPs of EBOV and MARV. a, Structural model of EEI-10 binding to GPCl. Amino residues (in yellow) binding to EEl-10 (in green) are shown as sticks. The oxygen, nitrogen, and hydrogen atoms are colored in red, blue, and white, respectively. For clarity, only polar hydrogen atoms are shown. The PDB ID of the EBOV-GPcl crystal structure used for molecular modeling is 5F1B. $b$, Inhibition of the T83A and I113A GP mutants by EEl-10. Luciferase activities were measured and 
normalized to those of untreated controls. Data shown are the average of three independent experiments, presented in mean \pm SD. c, Inhibition of MARV GP-pseudovirions by EEl-10 of different doses. d, Different doses of EEl-10 were tested for inhibition of MARV infection in Vero E6 cells. EC50 values were calculated with GraphPad Prism 5.0. Each data point is the average of three independent experiments. e, Superposition of the structures of the EBOV-GPCl/EEl-10 and MARV-GP1/EEl-10 complexes. EBOV-GPCl and MARV-GP1 are shown as yellow and slate cartoons, and their associate inhibitor EEI-10 was shown as green and orange sticks. f, Comparison of protein-ligand interactions in the EBOV-GPCl/EEl-10 and MARV-GP1/EEI-10 complexes. Key residues in the MARV-GP1/EEI-10 binding pocket are highlighted in red. The PDB IDs of crystal structures used for molecular modeling are 5F1B for EBOV GPcl and 5UQY for MARV GP1. 
a
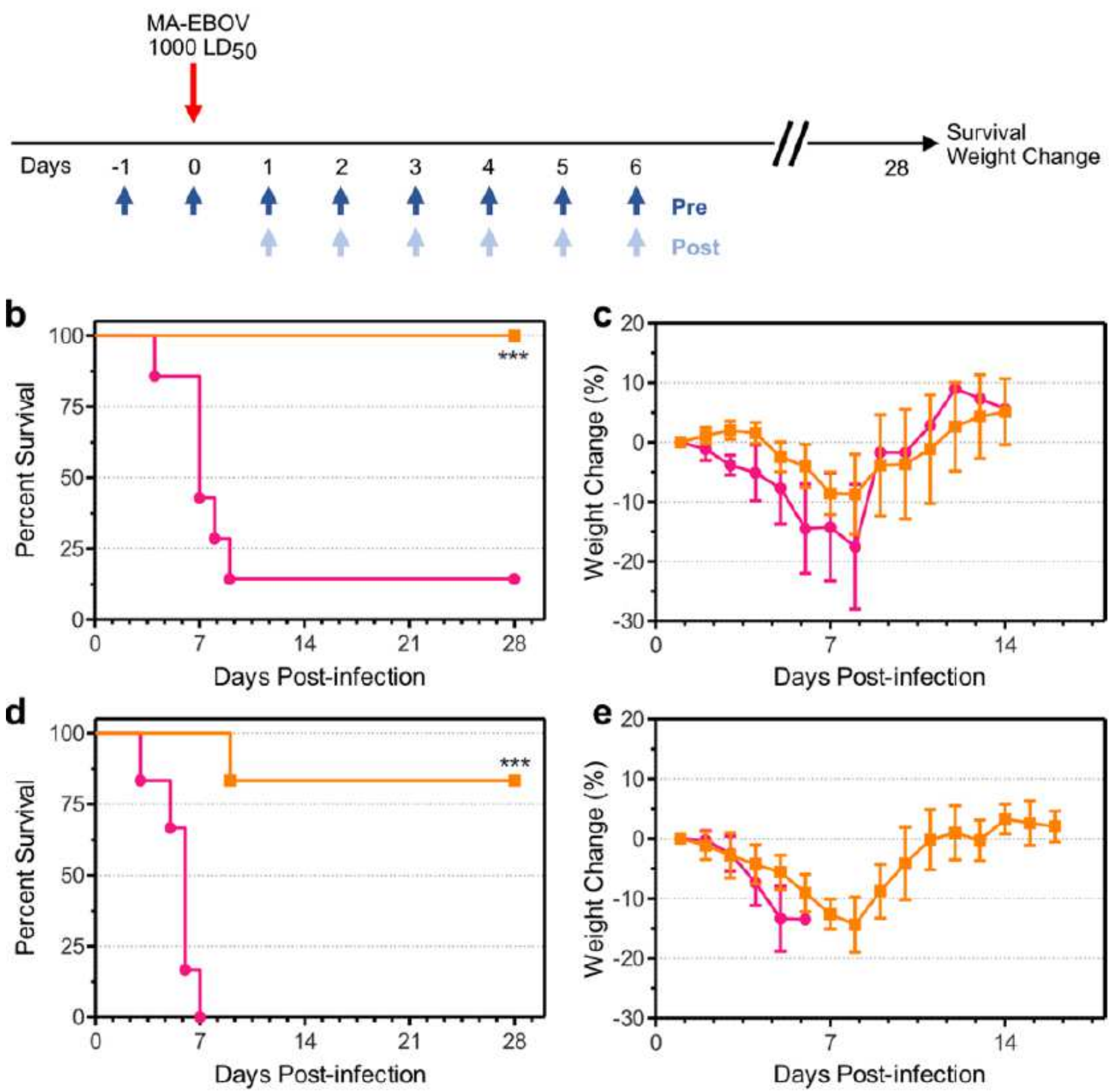

\section{$\mathrm{EEI}-10 \rightarrow$ PBS}

\section{Figure 5}

EEl-10 prevents lethal EBOV infection in mice. a, Schematic representation of EEl-10 treatment protocol and EBOV infection of mice. b,c, Balb/c mice were treated orally with EEl-10 (100 mg/kg; $\mathrm{n}=10)$ or PBS $(n=7)$ one day before infection with 1000 LD50 mouse-adapted EBOV (MA-EBOV). Treatment continued once daily until day 6 after infection. Survival (in b) and average percent weight change \pm standard deviation (in c) are shown. d,e, Balb/c mice were infected with 1000 LD50 MA-EBOV one day prior to oral 
treatment with EEI-10 $(100 \mathrm{mg} / \mathrm{kg} ; \mathrm{n}=6)$ or PBS $(\mathrm{n}=6)$. Treatment continued once daily until day 6 postinfection. Survival (in d) and average percent weight change \pm standard deviation (in e) are shown Survival curves were compared using the Log-rank Test; $* \star \star, p \leq 0.001$. 